TRANSACTIONS OF THE

AMERICAN MATHEMATICAL SOCIETY

Volume 356, Number 10, Pages 3963-3983

S 0002-9947(04)03602-5

Article electronically published on May 10, 2004

\title{
A MODIFIED BRAUER ALGEBRA AS CENTRALIZER ALGEBRA OF THE UNITARY GROUP
}

\author{
ALBERTO ELDUQUE
}

\begin{abstract}
The centralizer algebra of the action of $U(n)$ on the real tensor powers $\otimes_{\mathbb{R}}^{r} V$ of its natural module, $V=\mathbb{C}^{n}$, is described by means of a modification in the multiplication of the signed Brauer algebras. The relationships of this algebra with the invariants for $U(n)$ and with the decomposition of $\otimes_{\mathbb{R}}^{r} V$ into irreducible submodules is considered.
\end{abstract}

\section{INTRODUCTION}

The motivation for this work comes from a paper by Gray and Hervella [7]: Let $(M, g, J)$ be an almost Hermitian manifold; that is, $M$ is a Riemannian manifold with Riemannian metric $g$, and endowed with an almost complex structure $J$. Let $\nabla$ be the Riemannian connection and $F$ the Kähler form: $F(X, Y)=g(J X, Y)$ for any $X, Y \in \chi(M)$ (the set of smooth vector fields). The tensor $G=\nabla F$ satisfies

$$
G(X, Y, Z)=-G(X, Z, Y)=-G(X, J Y, J Z)
$$

for any $X, Y, Z \in \chi(M)$. Therefore, at any point $p \in M, \alpha=G_{p}$ belongs to

$$
\begin{aligned}
W_{p}=\left\{\alpha \in M_{p}^{*} \otimes_{\mathbb{R}} M_{p}^{*} \otimes_{\mathbb{R}} M_{p}^{*}:\right. \\
\left.\alpha(x, y, z)=-\alpha(x, z, y)=-\alpha(x, J y, J z) \forall x, y, z \in M_{p}\right\},
\end{aligned}
$$

where $M_{p}$ denotes the tangent space at $p$ and $M_{p}^{*}$ denotes its dual (the cotangent space), and $M_{p}^{*} \otimes_{\mathbb{R}} M_{p}^{*} \otimes_{\mathbb{R}} M_{p}^{*}$ is identified naturally with the space of trilinear forms on $M_{p}$.

The classification of almost hermitian manifolds in [7] is based on the decomposition of $W_{p}$ into irreducible modules under the action of the unitary group $U(n)$. This is done by first providing four specific subspaces of $W_{p}$ (if the dimension of $M$ is not very small) and then showing that they are irreducible (by means of invariants) and $W_{p}$ is their direct sum. No clue is given about how these four subspaces are obtained. A different way to obtain this decomposition is given in [5] based on complexification of $W_{p}$ and the use of Young symmetrizers.

Received by the editors June 9, 2003.

2000 Mathematics Subject Classification. Primary 20G05, 17B10.

Key words and phrases. Brauer algebra, unitary group, centralizer.

This research was supported by the Spanish Ministerio de Ciencia y Tecnología and FEDER (BFM 2001-3239-C03-03). 
The situation above extends naturally to the following problem:

Given a complex vector space $V$ of dimension $n$, endowed with a positive definite hermitian form $h: V \times V \rightarrow \mathbb{C}$, decompose the $r^{\text {th }}$ tensor power $\otimes_{\mathbb{R}}^{r}\left(V_{\mathbb{R}}\right)$ (over the real numbers!) into a direct sum of irreducible modules for the unitary group $U(V, h)=\{g \in G L(V): h(g v, g w)=h(v, w) \forall v, w \in V\}$.

Here, the convention is that $h(\alpha v, w)=\alpha h(v, w)$ and $h(v, w)=\overline{h(w, v)}$ for any $\alpha \in \mathbb{C}, v, w \in V$. Also, any complex vector space $V$ becomes a real vector space by restriction of scalars, denoted by $V_{\mathbb{R}}$. This explains the somehow redundant notation in the previous paragraph, which nonetheless will be useful to avoid ambiguities in what follows. For example, $S^{2}(V)_{\mathbb{R}}$ is the space of symmetric tensors in $V \otimes_{\mathbb{C}} V$, considered as a real vector space by restriction of scalars, while $S^{2}\left(V_{\mathbb{R}}\right)$ denotes the space of symmetric tensors in $\left(V_{\mathbb{R}}\right) \otimes_{\mathbb{R}}\left(V_{\mathbb{R}}\right)$. If $\operatorname{dim} V=n$, then $\operatorname{dim} V_{\mathbb{R}}=2 n$, $\operatorname{dim} S^{2}(V)_{\mathbb{R}}=2\left(\begin{array}{c}n+1 \\ 2\end{array}\right)=n(n+1)$ and $\operatorname{dim} S^{2}\left(V_{\mathbb{R}}\right)=\left(\begin{array}{c}2 n+1 \\ 2\end{array}\right)=n(2 n+1)$. In the same vein, $V^{*}$ denotes the dual vector space of the complex vector space $V$, so $V^{*}=\operatorname{Hom}_{\mathbb{C}}(V, \mathbb{C}),\left(V^{*}\right)_{\mathbb{R}}$ is the associated real vector space obtained by restriction of scalars, while $\left(V_{\mathbb{R}}\right)^{*}$ denotes the dual of the real vector space $V_{\mathbb{R}}$, so $\left(V_{\mathbb{R}}\right)^{*}=$ $\operatorname{Hom}_{\mathbb{R}}\left(V_{\mathbb{R}}, \mathbb{R}\right)$.

The well-known Schur-Weyl duality [16, 17, 20] relates the representation theory of the general linear group $G L(V)$ with that of the symmetric group $S_{r}$ via the naturally centralizing actions of the two groups on the space $\otimes_{\mathbb{C}}^{r} V: G L(V) \rightarrow$ $\otimes_{\mathbb{C}}^{r} V \leftarrow S_{r}$. Brauer [4] considered the analogous situation for the orthogonal and symplectic groups, where $S_{r}$ has to be replaced by what are now called the Brauer algebras: $O(V) \rightarrow \otimes_{\mathbb{C}}^{r} V \leftarrow \mathrm{Br}_{r}(n)$ and $S p(V) \rightarrow \otimes_{\mathbb{C}}^{r} V \leftarrow \operatorname{Br}_{r}(-n)$. More recently, Brauer algebras and their generalizations, specially the BMW algebra, have been looked at in the context of quantum groups and low-dimensional topology [11, 3 . 13, 8, 12.

In our problem, the decomposition of $\otimes_{\mathbb{R}}^{r}\left(V_{\mathbb{R}}\right)$ into a direct sum of irreducible modules for $U(V, h)$ is intimately related to the action of the centralizer algebra $\operatorname{End}_{U(V, h)}\left(\otimes_{\mathbb{R}}^{r}\left(V_{\mathbb{R}}\right)\right)$, and the main part of the paper will be devoted to computing this centralizer algebra. This will be done, following a classical approach, by relating it to the multilinear $U(V, h)$-invariant maps $f: V_{\mathbb{R}} \times \stackrel{r}{r} \times V_{\mathbb{R}} \rightarrow \mathbb{R}$. These invariants will be the subject of Section 2. Section 3 will be devoted to the determination of the centralizer algebra, while Section 4 will give a combinatorial description of it, as well as a presentation by generators and relations. It will turn out that the centralizer algebra looks like the Signed Brauer Algebra considered in [14, 15] (see Remark 4.8). This algebra appears, for sufficiently large dimension, as the centralizer algebra of the action of the product of orthogonal groups $O\left(S^{2}(V)\right) \times$ $O\left(\Lambda^{2}(V)\right)$ on $\otimes_{\mathbb{C}}^{r}\left(V \otimes_{\mathbb{C}} V\right)=\otimes_{\mathbb{C}}^{r}\left(S^{2}(V) \oplus \Lambda^{2}(V)\right)$, for a vector space $V$ equipped with a nondegenerate symmetric bilinear form $b: V \times V \rightarrow \mathbb{C}$, which induces a nondegenerate bilinear form on $V \otimes_{\mathbb{C}} V=S^{2}(V) \oplus \Lambda^{2}(V)$ (orthogonal direct sum). In Section 5 it will be shown how to use the information on the centralizer algebra, together with the results in [2], to decompose $\otimes_{\mathbb{R}}^{r}\left(V_{\mathbb{R}}\right)$ into a direct sum of irreducible $U(V, h)$-modules. A couple of examples will be given: the one in [7] mentioned above, and another one considered in [1], used to classify homogeneous Kähler structures.

Most of the results remain valid if 'positive definite' is weakened to 'nondegenerate', so they will be stated in this greater generality. 


\section{INVARIANTS}

This section is devoted to prove the next result:

Theorem 2.1. Let $V$ be an $n$-dimensional complex vector space, endowed with a nondegenerate hermitian form $h: V \times V \rightarrow \mathbb{C}$ and let $r \in \mathbb{N}$. If $f: V_{\mathbb{R}} \times \stackrel{r}{\cdots} \times V_{\mathbb{R}} \rightarrow$ $\mathbb{R}$ is a nonzero multilinear $U(V, h)$-invariant form, then $r$ is even $(r=2 m)$ and $f$ is a linear combination of the invariant maps

$$
\begin{aligned}
& V_{\mathbb{R}} \times \cdots \times V_{\mathbb{R}} \rightarrow \mathbb{R}, \\
& \left(v_{1}, \ldots, v_{2 m}\right) \mapsto \prod_{l=1}^{m}\left\langle v_{\sigma(2 l-1)}, J^{\delta_{l}} v_{\sigma(2 l)}\right\rangle,
\end{aligned}
$$

where $\sigma \in S_{2 m}$ (the symmetric group on $\left.\{1, \ldots, 2 m\}\right), \delta_{1}, \ldots, \delta_{m} \in\{0,1\}, J$ : $V_{\mathbb{R}} \rightarrow V_{\mathbb{R}}$ is the multiplication by $i \in \mathbb{C}\left(i^{2}=-1\right)$, and $\langle\mid\rangle$ denotes the real part of $h($ so that $h(v, w)=\langle v \mid w\rangle+i\langle v \mid J w\rangle$ for any $v, w \in V)$.

In case $\operatorname{dim} V \geq r$, this appears in [10]. For arbitrary $r$, it is asserted in [7] without proof. A proof will be provided here, which will be based on methods to be used later on.

Throughout the paper $(V, h), J$ and $\langle\mid\rangle$ will be assumed to satisfy the hypotheses of Theorem 2.1.

Let $r \in \mathbb{N}$, for any $l \in\{1, \ldots, r\}$ consider the $\mathbb{R}$-linear map

$$
\begin{aligned}
J_{l}: \otimes_{\mathbb{R}}^{r} V & \longrightarrow \otimes_{\mathbb{R}}^{r} V, \\
v_{1} \otimes \cdots \otimes v_{r} & \mapsto v_{1} \otimes \cdots \otimes J v_{l} \otimes \cdots \otimes v_{r}
\end{aligned}
$$

(action of $J$ on the $l^{\text {th }}$-spot). Then $J_{l} \in \operatorname{End}_{U(V, h)}\left(\otimes_{\mathbb{R}}^{r}\left(V_{\mathbb{R}}\right)\right)$, the centralizer algebra.

As a general rule, the elements of the centralizer algebra will act on the right.

Let $\mathcal{J}=\operatorname{alg}_{\mathbb{R}}\left\{J_{1}, \ldots, J_{r}\right\}$ be the (real) subalgebra of $\operatorname{End}_{U(V, h)}\left(\otimes_{\mathbb{R}}^{r}\left(V_{\mathbb{R}}\right)\right)$ generated by the $J_{l}$ 's. It is clear that $\mathcal{J}$ is isomorphic, as an algebra, to $\otimes_{\mathbb{R}}^{r} \mathbb{C}$, under the map that sends $J_{l}$ to $1 \otimes \cdots \otimes i \otimes \cdots \otimes 1$ ( $i$ in the $l^{\text {th }}$ slot) for any $l$. Note that for any $1 \leq l \neq m \leq r, \frac{1}{2}\left(1 \pm J_{l} J_{m}\right)$ is an idempotent in $\mathcal{J}$ since $J_{l}^{2}=-1$ for any l. For any nonempty subset $\mathcal{P} \subseteq\{1, \ldots, r\}$ and any $p \in \mathcal{P}$, let $\mathcal{P}^{c}=\{1, \ldots, r\} \backslash \mathcal{P}$ and consider the following element of $\mathcal{J}$ :

$$
e_{\mathcal{P}}=\frac{1}{2^{r-1}} \prod_{p \neq q \in \mathcal{P}}\left(1-J_{p} J_{q}\right) \prod_{q \in \mathcal{P} c}\left(1+J_{p} J_{q}\right) .
$$

Then:

Proposition 2.2. Under the conditions above:

(1) $e_{\mathcal{P}}$ does not depend on the chosen element $p \in \mathcal{P}$.

(2) $e_{\mathcal{P}}$ is a primitive idempotent of $\mathcal{J}$.

(3) Given any $p \in\{1, \ldots, r\}, \mathcal{J}=\oplus_{p \in \mathcal{P} \subseteq\{1, \ldots, r\}} \mathbb{C} e_{\mathcal{P}}$.

Proof. The $\mathbb{R}$-linear map $\mathbb{C} \otimes_{\mathbb{R}} \mathbb{C} \rightarrow \mathbb{C} \oplus \mathbb{C}, \alpha \otimes \beta \mapsto(\alpha \beta, \alpha \bar{\beta})$, yields an algebra isomorphism. Therefore, as real algebras, $\mathcal{J} \cong \otimes_{\mathbb{R}}^{r} \mathbb{C} \cong \mathbb{C}^{2^{r-1}}$. Now, fix $p \in \mathcal{P} \subseteq$ $\{1, \ldots, r\}$; then if $p \in \mathcal{P}^{\prime} \subseteq\{1, \ldots, r\}$ and $q \in \mathcal{P} \backslash \mathcal{P}^{\prime}, e_{\mathcal{P}} e_{\mathcal{P}^{\prime}}$ contains the factor $\left(1-J_{p} J_{q}\right)\left(1+J_{p} J_{q}\right)=0$, and hence $e_{\mathcal{P}} e_{\mathcal{P}^{\prime}}=0$. The same argument works for any $q \in \mathcal{P}^{\prime} \backslash \mathcal{P}$. Therefore $e_{\mathcal{P}}$ and $e_{\mathcal{P}^{\prime}}$ are orthogonal idempotents. Since there 
are $2^{r-1}$ subsets $\mathcal{P} \subseteq\{1, \ldots, r\}$ containing $p$ and $\mathcal{J}$ is an algebra over $\mathbb{C}$, where the action of $\mathbb{C}$ is given "on the $p^{\text {th }}$ slot" $\left(\mathcal{J} \cong \otimes_{\mathbb{R}}^{r} \mathbb{C}\right)$, to prove $(2)$ and (3) it is enough to check that $e_{\mathcal{P}}$ is nonzero for any $p \in \mathcal{P} \subseteq\{1, \ldots, r\}$. For simplicity, and without loss of generality, assume $p=1$. Then $2^{r-1} e_{\mathcal{P}}$ is the sum of $2^{r-1}$ summands $\pm J_{1}^{m} J_{2}^{\delta_{2}} \cdots J_{r}^{\delta_{r}}$, with $\delta_{l}=0$ or 1 , for $l=2, \ldots, r$, and $m=\delta_{2}+\cdots+\delta_{r}$. All these summands are linearly independent (over $\mathbb{R}$ ) in $\mathcal{J} \cong \otimes_{\mathbb{R}}^{r} \mathbb{C}$, so that $e_{\mathcal{P}} \neq 0$.

It remains for $(1)$ to be proved. Take $p \neq p^{\prime} \in \mathcal{P}$,

$$
e_{\mathcal{P}}=\frac{1}{2^{r-1}} \prod_{p \neq q \in \mathcal{P}}\left(1-J_{p} J_{q}\right) \prod_{q \in \mathcal{P}^{c}}\left(1+J_{p} J_{q}\right)
$$

and

$$
e_{\mathcal{P}}^{\prime}=\frac{1}{2^{r-1}} \prod_{p^{\prime} \neq q \in \mathcal{P}}\left(1-J_{p^{\prime}} J_{q}\right) \prod_{q \in \mathcal{P}^{c}}\left(1+J_{p^{\prime}} J_{q}\right) .
$$

Notice that for any $s \neq t$ in $\{1, \ldots, r\}$

$$
\begin{gathered}
\left(1-J_{s} J_{t}\right) J_{s}=J_{s}+J_{t}=\left(1-J_{s} J_{t}\right) J_{t}, \\
\left(1+J_{s} J_{t}\right) J_{s}=J_{s}-J_{t}=-\left(1+J_{s} J_{t}\right) J_{t}
\end{gathered}
$$

and hence

$$
\begin{cases}e_{\mathcal{P}} J_{p}=e_{\mathcal{P}} J_{q} & \text { for any } q \in \mathcal{P}, \\ e_{\mathcal{P}} J_{p}=-e_{\mathcal{P}} J_{q} & \text { for any } q \in \mathcal{P}^{c} .\end{cases}
$$

Therefore, since $J_{p}^{2}=-1$,

$$
e_{\mathcal{P}}\left(1-J_{s} J_{t}\right)= \begin{cases}0 & \text { if either } s \in \mathcal{P}, t \in \mathcal{P}^{c} \text { or } s \in \mathcal{P}^{c}, t \in \mathcal{P} \\ 2 e_{\mathcal{P}} & \text { if either } s, t \in \mathcal{P} \text { or } s, t \in \mathcal{P}^{c}\end{cases}
$$

while

$$
e_{\mathcal{P}}\left(1+J_{s} J_{t}\right)= \begin{cases}0 & \text { if either } s, t \in \mathcal{P} \text { or } s, t \in \mathcal{P}^{c}, \\ 2 e_{\mathcal{P}} & \text { if either } s \in \mathcal{P}, t \in \mathcal{P}^{c} \text { or } s \in \mathcal{P}^{c}, t \in \mathcal{P} .\end{cases}
$$

Therefore $e_{\mathcal{P}} e_{\mathcal{P}}^{\prime}=e_{\mathcal{P}}$ and, with the same argument, $e_{\mathcal{P}} e_{\mathcal{P}}^{\prime}=e_{\mathcal{P}}^{\prime}$, whence $e_{\mathcal{P}}=$ $e_{\mathcal{P}}^{\prime}$

Corollary 2.4. For any fixed $1 \leq p \leq r, 1=\sum_{p \in \mathcal{P} \subseteq\{1, \ldots, r\}} e_{\mathcal{P}}$.

Also, equation (2.3) immediately yields:

Lemma 2.5. Let $\emptyset \neq \mathcal{P} \subseteq\{1, \ldots, r\}$ and $p \in \mathcal{P}$. Then

$$
\left(\otimes_{\mathbb{R}}^{r}\left(V_{\mathbb{R}}\right)\right) e_{\mathcal{P}}=\left\{x \in \otimes_{\mathbb{R}}^{r}\left(V_{\mathbb{R}}\right): x J_{q}=x J_{p} \forall q \in \mathcal{P}, x J_{q}=-x J_{p} \forall q \in \mathcal{P}^{c}\right\} .
$$

The dual vector space $V^{*}$ is also a module for the unitary group $U(V, h)$. Take $\emptyset \neq \mathcal{P} \subseteq\{1, \ldots, r\}$ and consider

$$
V_{l}= \begin{cases}V & \text { if } l \in \mathcal{P} \\ V^{*} & \text { if } l \in \mathcal{P}^{c}\end{cases}
$$


Then:

Proposition 2.6. The $\mathbb{R}$-linear map

$$
\begin{gathered}
\Phi_{\mathcal{P}}:\left(\otimes_{\mathbb{R}}^{r}\left(V_{\mathbb{R}}\right)\right) e_{\mathcal{P}} \longrightarrow\left(V_{1} \otimes_{\mathbb{C}} V_{2} \otimes_{\mathbb{C}} \cdots \otimes_{\mathbb{C}} V_{r}\right)_{\mathbb{R}}, \\
\left(v_{1} \otimes \cdots \otimes v_{r}\right) e_{\mathcal{P}} \mapsto w_{1} \otimes w_{2} \otimes \cdots \otimes w_{r}
\end{gathered}
$$

where $w_{l}=v_{l}$ if $l \in \mathcal{P}$ and $w_{l}=h\left(-, v_{l}\right) \in V^{*}$ if $l \in \mathcal{P}^{c}$, is well defined and an isomorphism of $U(V, h)$-modules.

Proof. The linear map

$$
\begin{gathered}
\Psi_{\mathcal{P}}: \otimes_{\mathbb{R}}^{r}\left(V_{\mathbb{R}}\right) \longrightarrow\left(V_{1} \otimes_{\mathbb{C}} V_{2} \otimes_{\mathbb{C}} \cdots \otimes_{\mathbb{C}} V_{r}\right)_{\mathbb{R}}, \\
v_{1} \otimes \cdots \otimes v_{r} \mapsto w_{1} \otimes w_{2} \otimes \cdots \otimes w_{r}
\end{gathered}
$$

with $w_{1}, \ldots, w_{r}$ as above, is well defined and a homomorphism of $U(V, h)$-modules. Besides, if $p, q \in \mathcal{P}$, then $w_{p}=v_{p}$ and $w_{q}=v_{q}$ above, so that

$$
\begin{aligned}
\Psi_{\mathcal{P}}\left(\left(v_{1}\right.\right. & \left.\left.\otimes \cdots \otimes v_{r}\right) \frac{1}{2}\left(1-J_{p} J_{q}\right)\right) \\
& =\frac{1}{2} \Psi_{\mathcal{P}}\left(v_{1} \otimes \cdots \otimes v_{r}-v_{1} \otimes \cdots \otimes i v_{p} \otimes \cdots \otimes i v_{q} \otimes \cdots \otimes v_{r}\right) \\
& =\frac{1}{2}\left(w_{1} \otimes \cdots \otimes w_{r}-w_{1} \otimes \cdots \otimes i w_{p} \otimes \cdots \otimes i w_{q} \otimes \cdots \otimes w_{r}\right) \\
& =w_{1} \otimes \cdots \otimes w_{r}
\end{aligned}
$$

while if $p \in \mathcal{P}$ and $q \in \mathcal{P}^{c}$, then $w_{p}=v_{p}$ and $w_{q}=h\left(-, v_{q}\right)$ so, since $h\left(-, i v_{q}\right)=$ $-i h\left(-, v_{q}\right)=-i w_{q}$,

$$
\begin{aligned}
\Psi_{\mathcal{P}}\left(\left(v_{1}\right.\right. & \left.\left.\otimes \cdots \otimes v_{r}\right) \frac{1}{2}\left(1+J_{p} J_{q}\right)\right) \\
& =\frac{1}{2} \Psi_{\mathcal{P}}\left(v_{1} \otimes \cdots \otimes v_{r}+v_{1} \otimes \cdots \otimes i v_{p} \otimes \cdots \otimes i v_{q} \otimes \cdots \otimes v_{r}\right) \\
& =\frac{1}{2}\left(w_{1} \otimes \cdots \otimes w_{r}-w_{1} \otimes \cdots \otimes i w_{p} \otimes \cdots \otimes i w_{q} \otimes \cdots \otimes w_{r}\right) \\
& =w_{1} \otimes \cdots \otimes w_{r} .
\end{aligned}
$$

Therefore, since $e_{\mathcal{P}}=\prod_{p \neq q \in \mathcal{P}}\left(\frac{1}{2}\left(1-J_{p} J_{q}\right)\right) \prod_{q \in \mathcal{P}^{c}}\left(\frac{1}{2}\left(1+J_{p} J_{q}\right)\right)$, it follows that $\Psi_{\mathcal{P}}(x)=\Psi_{\mathcal{P}}\left(x e_{\mathcal{P}}\right)$ for any $x \in \otimes_{\mathbb{R}}^{r}\left(V_{\mathbb{R}}\right)$, and then $\Psi_{\mathcal{P}}$ restricts to $\Phi_{\mathcal{P}}$, which is thus well defined. The inverse is given by

$$
\begin{aligned}
\Phi_{\mathcal{P}}^{-1}:\left(V_{1} \otimes_{\mathbb{C}} \cdots \otimes_{\mathbb{C}} V_{r}\right)_{\mathbb{R}} & \longrightarrow\left(\otimes_{\mathbb{R}}^{r}\left(V_{\mathbb{R}}\right)\right) e_{\mathcal{P}}, \\
w_{1} \otimes \cdots \otimes w_{r} & \mapsto\left(v_{1} \otimes \cdots \otimes v_{p}\right) e_{\mathcal{P}},
\end{aligned}
$$

where $v_{l}=w_{l}$ if $l \in \mathcal{P}$, while $w_{l}=h\left(-, v_{l}\right)$ for a unique $v_{l} \in V$ if $l \in \mathcal{P}^{c}$. This is well defined because of Lemma 2.5

Now, Corollary 2.4 and Proposition 2.6 yield:

Corollary 2.8. Fix $p \in\{1, \ldots, r\}$. Then the $U(V, h)$-module $\otimes_{\mathbb{R}}^{r}\left(V_{\mathbb{R}}\right)$ is isomorphic to

$$
\bigoplus_{p \in \mathcal{P} \subseteq\{1, \ldots, r\}}\left(V_{1 \mathcal{P}} \otimes_{\mathbb{C}} \cdots \otimes_{\mathbb{C}} V_{r \mathcal{P}}\right)_{\mathbb{R}},
$$

where $V_{l \mathcal{P}}=V$ if $l \in \mathcal{P}$, while $V_{l \mathcal{P}}=V^{*}$ otherwise. 
The real vector space $\otimes_{\mathbb{R}}^{r}\left(V_{\mathbb{R}}\right)$ is a complex vector space with the natural action of $\mathbb{C}$ on the $p^{\text {th }}$ slot. This complex vector space will be denoted by $\left[\otimes_{\mathbb{R}}^{r}\left(V_{\mathbb{R}}\right)\right]_{\mathbb{C}, p}$. The isomorphism in Corollary 2.8 is then an isomorphism of complex vector spaces too:

$$
\left[\otimes_{\mathbb{R}}^{r}\left(V_{\mathbb{R}}\right)\right]_{\mathbb{C}, p} \cong \bigoplus_{p \in \mathcal{P} \subseteq\{1, \ldots, r\}} V_{1 \mathcal{P}} \otimes_{\mathbb{C}} \cdots \otimes_{\mathbb{C}} V_{r \mathcal{P}} .
$$

The final prerequisite in the proof of Theorem 2.1 is the next straightforward result:

Lemma 2.10. Let $G$ be a real Lie group, $\rho: G \rightarrow \operatorname{End}_{\mathbb{C}}(W)$ a complex representation of $G$, and $f: W_{\mathbb{R}} \rightarrow \mathbb{R}$ a linear $G$-invariant map $(f(x . w)=f(w)$ for any $x \in G, w \in W)$. Then there is a complex linear $G$-invariant map $g: W \rightarrow \mathbb{C}$ such that $f$ is the real part of $g(f=\operatorname{Re} g)$.

Proof of Theorem 2.1. From the previous results we obtain

$$
\begin{aligned}
& \left\{S: V_{\mathbb{R}} \times \stackrel{r}{r} \times V_{\mathbb{R}} \rightarrow \mathbb{R} \mid S \text { multilinear and } U(V, h) \text {-invariant }\right\} \\
& \quad \simeq\left\{S: \otimes_{\mathbb{R}}^{r}\left(V_{\mathbb{R}}\right) \rightarrow \mathbb{R} \mid S \text { linear and } U(V, h) \text {-invariant }\right\} \\
& \quad=\bigoplus_{1 \in \mathcal{P} \subseteq\{1, \ldots, r\}}\left\{S_{\mathcal{P}}:\left(\otimes_{\mathbb{R}}^{r}\left(V_{\mathbb{R}}\right)\right) e_{\mathcal{P}} \rightarrow \mathbb{R} \mid S_{\mathcal{P}} \text { linear and } U(V, h) \text {-invariant }\right\} \\
& \quad \simeq \bigoplus_{1 \in \mathcal{P} \subseteq\{1, \ldots, r\}}\left\{S_{\mathcal{P}}:\left(V_{1 \mathcal{P}} \otimes_{\mathbb{C}} \cdots \otimes_{\mathbb{C}} V_{r \mathcal{P}}\right)_{\mathbb{R}} \rightarrow \mathbb{R} \mid S_{\mathcal{P}} \text { linear and } U(V, h) \text {-invariant }\right\} \\
& \quad=\bigoplus_{1 \in \mathcal{P} \subseteq\{1, \ldots, r\}}\left\{\operatorname{Re} T_{\mathcal{P}} \mid T_{\mathcal{P}}: V_{1 \mathcal{P}} \otimes_{\mathbb{C}} \cdots \otimes_{\mathbb{C}} V_{r \mathcal{P}} \rightarrow \mathbb{C}(\mathbb{C} \text { - }) \text { linear and } U(V, h) \text {-invariant }\right\} \\
& \quad=\bigoplus_{1 \in \mathcal{P} \subseteq\{1, \ldots, r\}}\left\{\operatorname{Re} T_{\mathcal{P}} \mid T_{\mathcal{P}}: V_{1 \mathcal{P}} \otimes_{\mathbb{C}} \cdots \otimes_{\mathbb{C}} V_{r \mathcal{P}} \rightarrow \mathbb{C}(\mathbb{C} \text { - }) \text { linear and } G L(V) \text {-invariant }\right\} .
\end{aligned}
$$

(The last equality is due to the fact that $U(V, h)$ is a form of $G L(V)$.) But the invariant theory of $G L(V)$ [20] shows that

$$
\left\{T: V_{1 \mathcal{P}} \otimes_{\mathbb{C}} \cdots \otimes_{\mathbb{C}} V_{r \mathcal{P}} \rightarrow \mathbb{C} \mid T \text { is } \mathbb{C} \text {-linear and } G L(V) \text {-invariant }\right\}
$$

is trivial unless $r$ is even, $r=2 m$, and $\mathcal{P}$ contains exactly $m$ elements. In this latter case, $\mathcal{P}=\left\{l_{1}, \ldots, l_{m}\right\}\left(l_{1}=1\right), \mathcal{P}^{c}=\left\{s_{1}, \ldots, s_{m}\right\}$ and any such invariant $T$ is a (complex) linear combination of invariants of the form

$$
w_{1} \otimes \cdots \otimes w_{2 m} \mapsto \prod_{j=1}^{m} \varphi_{s_{\sigma(j)}}\left(v_{l_{j}}\right),
$$

where $w_{l}=v_{l} \in V$ for $l \in \mathcal{P}, w_{l}=\varphi_{l} \in V^{*}$ for $l \in \mathcal{P}^{c}$, and $\sigma \in S_{m}$, the symmetric group on $\{1, \ldots, m\}$.

Taking into account the definitions of the isomorphisms $\Phi_{\mathcal{P}}$ and homomorphisms $\Psi_{\mathcal{P}}$ in Proposition 2.6 and (2.7), any $U(V, h)$-invariant linear map $T:\left[\otimes_{\mathbb{R}}^{r}\left(V_{\mathbb{R}}\right)\right]_{\mathbb{C}, 1} \rightarrow$ $\mathbb{C}$ is a complex linear combination of the maps

$$
v_{1} \otimes \cdots \otimes v_{2 m} \mapsto \prod_{j=1}^{m} h\left(v_{\sigma(2 j-1)}, v_{\sigma(2 j)}\right),
$$

where $\sigma \in S_{2 m}$. Since $h(v, w)=\langle v \mid w\rangle+i\langle v \mid J w\rangle$, Lemma 2.10 finishes the proof. 
A final remark for this section is that using the invariant theory for $S L(V)$ instead of $G L(V)$ and the same arguments as above, one arrives at:

Proposition 2.11. The invariant multilinear $S U(V, h)$-invariant maps $f: V_{\mathbb{R}} \times \stackrel{r}{.}$ $\times V_{\mathbb{R}} \rightarrow \mathbb{R}$ are exactly the linear combinations of the maps

$$
\begin{aligned}
& \left(v_{1}, \ldots, v_{r}\right) \\
\mapsto & \left(\prod_{j=1}^{m}\left\langle v_{\sigma(2 j-1)}, J^{\delta_{j}} v_{\sigma(2 j)}\right\rangle\right)\left(\prod_{l=0}^{s-1} \operatorname{Re}\left(\operatorname{det}_{\mathbb{C}}\left(J^{\delta_{m+l+1}} v_{\sigma(2 m+n l+1)}, \cdots, v_{\sigma(2 m+n(l+1))}\right)\right)\right),
\end{aligned}
$$

where $n=\operatorname{dim} V, m, s \geq 0$ with $r=2 m+n s, \sigma \in S_{r}, \delta_{1}, \ldots, \delta_{m+s} \in\{0,1\}$ and $\operatorname{det}_{\mathbb{C}}: V \times \stackrel{r}{\cdots} \times V \rightarrow \mathbb{C}$ is any fixed nonzero alternating multilinear map.

For $n=1$ any multilinear map can be expressed in this form.

\section{Centralizer algebra}

The aim of this section is to compute the centralizer algebra of the action of $U(V, h)$ on $\otimes_{\mathbb{R}}^{r}\left(V_{\mathbb{R}}\right): \operatorname{End}_{U(V, h)}\left(\otimes_{\mathbb{R}}^{r}\left(V_{\mathbb{R}}\right)\right)$. As checked in the previous section, the $J_{l}$ 's belong to this algebra and also, for any $\sigma$ in the symmetric group $S_{r}$, so does the map $\rho(\sigma): v_{1} \otimes \cdots \otimes v_{r} \mapsto\left(v_{1} \otimes \cdots \otimes v_{r}\right) \sigma:=v_{\sigma(1)} \otimes \cdots \otimes v_{\sigma(r)}$. Let $\left\{e_{l}\right\}_{l=1}^{2 n}$ be a basis of $V_{\mathbb{R}}$, and let $\left\{f_{l}\right\}_{l=1}^{2 n}$ be its dual basis relative to $\langle\mid\rangle$ (so that $\left\langle e_{p} \mid f_{q}\right\rangle=\delta_{p q}$ for any $p, q)$. The contraction maps $c_{p q}(1 \leq p<q \leq r)$ defined as follows:

$$
\begin{aligned}
& \left(v_{1} \otimes \cdots \otimes v_{r}\right) c_{p q} \\
& =\left\langle v_{p} \mid v_{q}\right\rangle \sum_{a=1}^{2 n} v_{1} \otimes \cdots \otimes v_{p-1} \otimes e_{a} \otimes v_{p+1} \otimes \cdots \otimes v_{q-1} \otimes f_{a} \otimes v_{q+1} \cdots \otimes v_{r}
\end{aligned}
$$

also belong to $\operatorname{End}_{U(V, h)}\left(\otimes_{\mathbb{R}}^{r}\left(V_{\mathbb{R}}\right)\right)$. Notice that $c_{p q}$ does not depend on the chosen dual bases.

All these elements generate the centralizer algebra:

Theorem 3.2. The centralizer algebra $\operatorname{End}_{U(V, h)}\left(\otimes_{\mathbb{R}}^{r}\left(V_{\mathbb{R}}\right)\right.$ ) is generated (as a real algebra) by the $J_{l}$ 's, $c_{p q}$ 's and the action of the symmetric group $S_{r}$ :

$$
\operatorname{End}_{U(V, h)}\left(\otimes_{\mathbb{R}}^{r}\left(V_{\mathbb{R}}\right)\right)=\operatorname{alg}_{\mathbb{R}}\left\{\rho\left(S_{r}\right), J_{l}, c_{p q}: l, p, q=1, \ldots, r, p<q\right\} .
$$

Proof. To compute the centralizer algebra $\operatorname{End}_{U(V, h)}\left(\otimes_{\mathbb{R}}^{r}\left(V_{\mathbb{R}}\right)\right)$, it is enough to use the fact that $\operatorname{End}_{\mathbb{R}}\left(\otimes_{\mathbb{R}}^{r}\left(V_{\mathbb{R}}\right)\right)$ is isomorphic (as a vector space and as a $U(V, h)$ module) to $\left(\otimes_{\mathbb{R}}^{r}\left(V_{\mathbb{R}}\right)^{*}\right) \otimes_{\mathbb{R}}\left(\otimes_{\mathbb{R}}^{r}\left(V_{\mathbb{R}}\right)\right)$. But $V_{\mathbb{R}}$ is isomorphic to $\left(V_{\mathbb{R}}\right)^{*}$ as a $U(V, h)$ module by means of $\langle\mid\rangle\left(V_{\mathbb{R}} \rightarrow\left(V_{\mathbb{R}}\right)^{*}, v \mapsto\langle v \mid-\rangle\right.$, is an isomorphism). Therefore, $\operatorname{End}_{\mathbb{R}}\left(\otimes_{\mathbb{R}}^{r}\left(V_{\mathbb{R}}\right)\right)$ is isomorphic to $\otimes_{\mathbb{R}}^{2 r}\left(V_{\mathbb{R}}\right)^{*}$, which is naturally identified with the space of multilinear maps: $f: V_{\mathbb{R}} \times \cdots \times V_{\mathbb{R}} \rightarrow \mathbb{R}$. Under these isomorphisms, the centralizer algebra $\operatorname{End}_{U(V, h)}\left(\otimes_{\mathbb{R}}^{r}\left(V_{\mathbb{R}}\right)\right)$ (which is the subalgebra of $\operatorname{End}_{\mathbb{R}}\left(\otimes_{\mathbb{R}}^{r}\left(V_{\mathbb{R}}\right)\right.$ ) fixed under the action of $U(V, h))$ corresponds to the space of multilinear and $U(V, h)$-invariant maps.

Hence, to compute $\operatorname{End}_{U(V, h)}\left(\otimes_{\mathbb{R}}^{r}\left(V_{\mathbb{R}}\right)\right)$ one has just to keep track of the isomorphisms above. Let us proceed with an example: consider the multilinear $U(V, h)$ invariant map

$$
\begin{aligned}
f: V_{\mathbb{R}} \times V_{\mathbb{R}} \times V_{\mathbb{R}} \times V_{\mathbb{R}} \times V_{\mathbb{R}} \times V_{\mathbb{R}} & \longrightarrow \mathbb{R} \\
\left(v_{1}, v_{2}, v_{3}, v_{4}, v_{5}, v_{6}\right) & \mapsto\left\langle v_{1} \mid J v_{3}\right\rangle\left\langle v_{2} \mid J v_{4}\right\rangle\left\langle v_{5} \mid J v_{6}\right\rangle
\end{aligned}
$$


and take $\left\{e_{l}\right\}_{l=1}^{2 n}$ and $\left\{f_{l}\right\}_{l=1}^{2 n}$ dual bases relative to $\langle\mid\rangle$ as above. Let $e_{l}^{*}=\left\langle e_{l} \mid-\right\rangle$, $f_{l}^{*}=\left\langle f_{l} \mid-\right\rangle \in V_{\mathbb{R}}^{*}$ for any $l$. Then notice that the bilinear $U(V, h)$-invariant maps $(v, w) \mapsto\langle v \mid w\rangle$ and $(v, w) \mapsto\langle v \mid J w\rangle$ correspond in $\left(V_{\mathbb{R}}\right)^{*} \otimes_{\mathbb{R}}\left(V_{\mathbb{R}}\right)^{*}$ to $\sum_{l=1}^{2 n} e_{l}^{*} \otimes f_{l}^{*}$ and $\sum_{l=1}^{2 n} e_{l}^{*} \otimes\left(f_{l}^{*} \circ J\right)$, respectively. Thus, the multilinear map $f$ in (3.3) corresponds to

$$
\begin{aligned}
f & \simeq \sum_{a, b, c=1}^{2 n} e_{a}^{*} \otimes e_{b}^{*} \otimes\left(f_{a}^{*} \circ J\right) \otimes\left(f_{b}^{*} \circ J\right) \otimes e_{c}^{*} \otimes\left(f_{c}^{*} \circ J\right) \quad \text { in } \otimes_{\mathbb{R}}^{6}\left(V_{\mathbb{R}}\right)^{*} \\
& \simeq(-1)^{2} \sum_{a, b, c=1}^{2 n} e_{a}^{*} \otimes e_{b}^{*} \otimes\left(f_{a}^{*} \circ J\right) \otimes J f_{b} \otimes e_{c} \otimes J f_{c} \\
& \operatorname{in}\left(\otimes_{\mathbb{R}}^{3}\left(V_{\mathbb{R}}\right)^{*}\right) \otimes_{\mathbb{R}}\left(\otimes_{\mathbb{R}}^{3}\left(V_{\mathbb{R}}\right)\right)\left(\text { since }\left\langle f_{a} \mid J v\right\rangle=-\left\langle J f_{a} \mid v\right\rangle\right) \\
& \simeq\left(v_{1} \otimes v_{2} \otimes v_{3} \mapsto \sum_{a, b, c=1}^{2 n} e_{a}^{*}\left(v_{1}\right) e_{b}^{*}\left(v_{2}\right) f_{a}^{*}\left(J v_{3}\right) J f_{b} \otimes e_{c} \otimes J f_{c}\right) \\
& =\left(v_{1} \otimes v_{2} \otimes v_{3} \mapsto\left\langle v_{1} \mid J v_{3}\right\rangle \sum_{c=1}^{2 n}\left(\sum_{b=1}^{2 n} e_{b}^{*}\left(v_{2}\right) J f_{b}\right) \otimes e_{c} \otimes J f_{c}\right) \\
& =\left(v_{1} \otimes \otimes_{\mathbb{R}}\left(V_{\mathbb{R}}\right)\right) \\
& \left.=J_{3} c_{13} J_{2} J_{3} \rho((12)) \in v_{3} \mapsto\left\langle v_{1} \mid J v_{3}\right\rangle \sum_{c=1}^{2 n} J v_{2} \otimes e_{c} \otimes J f_{c}\right)
\end{aligned}
$$

where (12) denotes the permutation of the first two slots.

The result follows since the arguments used for this particular $f$ in (3.3) work in general.

\section{Combinatorial Description}

Consider the element in equation (3.4), which belongs to the centralizer algebra $\operatorname{End}_{U(V, h)}\left(\otimes_{\mathbb{R}}^{6}\left(V_{\mathbb{R}}\right)\right)$ :

$$
v_{1} \otimes v_{2} \otimes v_{3} \mapsto\left\langle v_{1} \mid J v_{3}\right\rangle \sum_{a=1}^{2 n} J v_{2} \otimes e_{a} \otimes J f_{a} .
$$

(Notation as in the previous section.) It will be represented by the marked diagram:

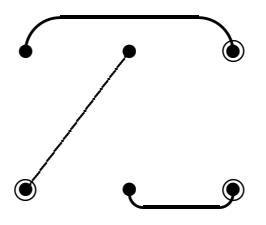


A marked diagram on $2 r$ vertices is a graph with $2 r$ vertices arranged in two rows of $r$ vertices each, one above the other, and $r$ edges such that each vertex is incident to precisely one edge. The rightmost vertex of each 'horizontal' edge (i.e., joining vertices in the same row) and the bottommost vertex of each 'vertical' edge (i.e., joining vertices in different rows) may (or may not) be 'marked'.

There are $(2 r-1)$ !! 'unmarked diagrams' and, therefore, $2^{r}((2 r-1) ! !)$ marked diagrams. The unmarked diagrams form a basis of the classical Brauer algebra.

Any such marked diagram represents an element of the centralizer algebra $\operatorname{End}_{U(V, h)}\left(\otimes_{\mathbb{R}}^{r}\left(V_{\mathbb{R}}\right)\right)$. For instance, the marked diagram

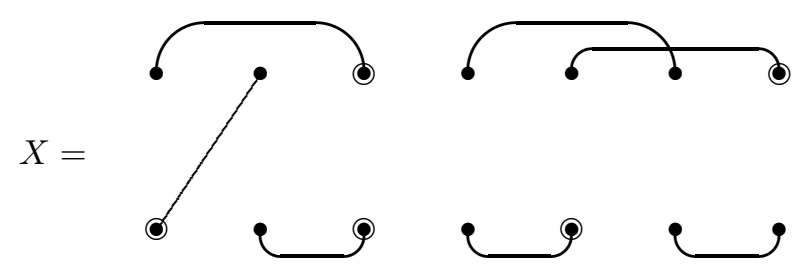

represents the map

$$
\begin{aligned}
\rho(X) & : v_{1} \otimes \cdots \otimes v_{7} \\
& \mapsto\left\langle v_{1} \mid J v_{3}\right\rangle\left\langle v_{4} \mid v_{6}\right\rangle\left\langle v_{5} \mid J v_{7}\right\rangle \sum_{a, b, c=1}^{2 n} J v_{2} \otimes e_{a} \otimes J f_{a} \otimes e_{b} \otimes J f_{b} \otimes e_{c} \otimes f_{c} .
\end{aligned}
$$

Let $\mathrm{D}_{\mathrm{r}}^{\text {marked }}$ denote the real vector space with a basis formed by the marked diagrams with $2 r$ vertices, numbered from 1 to $r$ from left to right in the top row and from $r+1$ to $2 r$ from left to right on the bottom row. The procedure above provides a map from the set of marked diagrams into $\operatorname{End}_{U(V, h)}\left(\otimes_{\mathbb{R}}^{r}\left(V_{\mathbb{R}}\right)\right)$ and hence a linear map

$$
\rho: \mathrm{D}_{\mathrm{r}}^{\text {marked }} \longrightarrow \operatorname{End}_{U(V, h)}\left(\otimes_{\mathbb{R}}^{r}\left(V_{\mathbb{R}}\right)\right)
$$

This linear map $\rho$ is onto because of Theorem 3.2 (or Theorem 2.1).

Proposition 4.1. $\rho$ is a bijection if and only if $n \geq r$.

Proof. Let $X$ be a marked diagram and let us split the edges in $X$ according to whether its rightmost or bottommost vertex is marked or not:

$$
\begin{aligned}
& X^{+}=\{(p, q) \text { edge in } X \mid p<q \text { and } q \text { is not marked }\} \\
& X^{-}=\{(p, q) \text { edge in } X \mid p<q \text { and } q \text { is marked }\} .
\end{aligned}
$$

Assume $X^{+}=\left\{\left(p_{1}, q_{1}\right), \ldots,\left(p_{s}, q_{s}\right)\right\}$ and $X^{-}=\left\{\left(p_{s+1}, q_{s+1}\right), \ldots,\left(p_{r}, q_{r}\right)\right\}$. Let $\left\{d_{l}\right\}_{l=1}^{n}$ be an $h$-orthogonal basis of $V$ (that is, $h\left(d_{l}, d_{m}\right)=0$ for $l \neq m$ ), so that $\left\{d_{1}, \ldots, d_{n}, J d_{1}, \ldots, J d_{n}\right\}$ is an orthogonal basis of $V_{\mathbb{R}}$ relative to $\langle\mid\rangle$. Through the natural isomorphisms considered in Section $3, \rho(X)$ corresponds to the multilinear invariant map:

$$
f_{X}:\left(v_{1}, \ldots, v_{2 r}\right) \mapsto \pm \prod_{(p, q) \in X^{+}}\left\langle v_{p} \mid v_{q}\right\rangle \prod_{(p, q) \in X^{-}}\left\langle v_{p} \mid J v_{q}\right\rangle
$$

(the \pm sign appears due to the skew symmetry of $J$ relative to $\langle\mid\rangle$ ).

If $n \geq r$, take $v_{p_{l}}=d_{l}=v_{q_{l}}$ for $l=1, \ldots, s$ and $v_{p_{l}}=J d_{l}, v_{q_{l}}=d_{l}$ for $l=s+1, \ldots, r$. Then $f_{X}\left(v_{1}, \ldots, v_{2 r}\right) \neq 0$, while $f_{Y}\left(v_{1}, \ldots, v_{2 r}\right)=0$ for any 
$Y \neq X$, due to the orthogonality of the chosen basis. This shows that for $n \geq r, \rho$ is one-to-one, and hence a bijection.

However, if $n \leq r-1$, consider the element $z \in \operatorname{End}_{U(V, h)}\left(\otimes_{\mathbb{R}}^{r}\left(V_{\mathbb{R}}\right)\right)$ given by

$$
z=\left(1-J_{1} J_{2}\right)\left(1-J_{1} J_{3}\right) \cdots\left(1-J_{1} J_{r}\right)\left(\sum_{\sigma \in S_{r}}(-1)^{\sigma} \rho\left(\sigma_{r}\right)\right),
$$

where $(-1)^{\sigma}$ denotes the signature of $\sigma$. Notice that

$$
z=2^{r-1} e_{\{1, \ldots, r\}}\left(\sum_{\sigma \in S_{r}}(-1)^{\sigma} \rho\left(\sigma_{r}\right)\right) .
$$

When expanded, $z$ appears as the image under $\rho$ of a nontrivial linear combination of different marked diagrams without horizontal edges. For any $\sigma \in S_{r}$ and any $l \in\{1, \ldots, r\}, \rho(\sigma) J_{l}=J_{\sigma(l)} \rho(\sigma)$ (remember that $\operatorname{End}_{U(V, h)}\left(\otimes_{\mathbb{R}}^{r}\left(V_{\mathbb{R}}\right)\right)$ acts on the right) so, due to Proposition [2.2 1 , for any $\sigma \in S_{r}$ one has $e_{\{1, \ldots, r\}} \rho(\sigma)=$ $\rho(\sigma) e_{\{1, \ldots, r\}}$. Hence the isomorphism

$$
\begin{aligned}
\Phi_{\{1, \ldots, r\}}:\left(\otimes_{\mathbb{R}}^{r}\left(V_{\mathbb{R}}\right)\right) e_{\{1, \ldots, r\}} & \longrightarrow\left(\otimes_{\mathbb{C}}^{r} V\right)_{\mathbb{R}}, \\
\left(v_{1} \otimes \cdots \otimes v_{r}\right) e_{\{1, \ldots, r\}} & \mapsto v_{1} \otimes \cdots \otimes v_{r},
\end{aligned}
$$

given in Proposition 2.6, preserves the action of $S_{r}$. Since $n \geq r-1, \sum_{\sigma \in S_{r}}(-1)^{\sigma} \sigma$ acts trivially on $\otimes_{\mathbb{C}}^{r} V$ and, therefore, $z$ in (4.2) acts trivially on $\otimes_{\mathbb{R}}^{r}\left(V_{\mathbb{R}}\right)$. That is, $z=0$. Thus $\rho$ is not one-to-one in this case.

The multiplication (composition of maps) in $\operatorname{End}_{U(V, h)}\left(\otimes_{\mathbb{R}}^{r}\left(V_{\mathbb{R}}\right)\right)$ can be lifted to a multiplication in $\mathrm{D}_{\mathrm{r}}^{\text {marked }}$. Let us look at an example first. Take the following two marked diagrams:
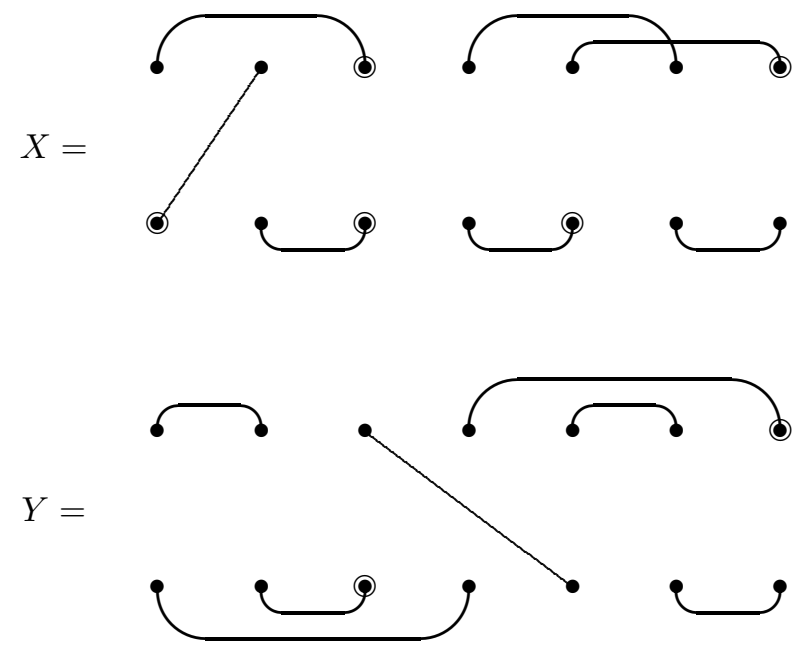

Then:

$$
\begin{aligned}
& v_{1} \otimes \cdots \otimes v_{7} \\
& \stackrel{\rho_{X}}{\longrightarrow}\left\langle v_{1} \mid J v_{3}\right\rangle\left\langle v_{4} \mid v_{6}\right\rangle\left\langle v_{5} \mid J v_{7}\right\rangle \sum_{a, b, c=1}^{2 n} J v_{2} \otimes e_{a} \otimes J f_{a} \otimes e_{b} \otimes J f_{b} \otimes e_{c} \otimes f_{c}
\end{aligned}
$$


(with notations already familiar) and

$$
\begin{aligned}
& J v_{2} \otimes e_{a} \otimes J f_{a} \otimes e_{b} \otimes J f_{b} \otimes e_{c} \otimes f_{c} \\
& \stackrel{\rho_{Y}}{\longrightarrow}\left\langle J v_{2} \mid e_{a}\right\rangle\left\langle e_{b} \mid J f_{c}\right\rangle\left\langle J f_{b} \mid e_{c}\right\rangle \sum_{j, k, l=1}^{2 n} e_{j} \otimes e_{k} \otimes J f_{k} \otimes f_{j} \otimes J f_{a} \otimes e_{l} \otimes f_{l}
\end{aligned}
$$

but $\sum_{a=1}^{2 n}\left\langle J v_{2} \mid e_{a}\right\rangle J f_{a}=J\left(\sum_{a=1}^{2 n}\left\langle J v_{2} \mid e_{a}\right\rangle f_{a}\right)=J\left(J v_{2}\right)=-v_{2}$, and

$$
\begin{aligned}
& \sum_{b, c=1}^{2 n}\left\langle e_{b} \mid J f_{c}\right\rangle\left\langle J f_{b} \mid e_{c}\right\rangle=-\sum_{b, c=1}^{2 n}\left\langle e_{b} \mid J f_{c}\right\rangle\left\langle f_{b} \mid J e_{c}\right\rangle \\
& =-\sum_{c=1}^{2 n}\left\langle\sum_{b=1}^{2 n}\left\langle e_{b} \mid J f_{c}\right\rangle f_{b} \mid J e_{c}\right\rangle=-\sum_{c=1}^{2 n}\left\langle J f_{c} \mid J e_{c}\right\rangle=-\sum_{c=1}^{2 n}\left\langle e_{c} \mid f_{c}\right\rangle=-2 n,
\end{aligned}
$$

since $J$ is skew-symmetric relative to $\langle\mid\rangle$ and $J^{2}=-1$. Therefore,

$$
\begin{aligned}
& v_{1} \otimes \cdots \otimes v_{7} \\
& \stackrel{\rho_{X} \rho_{Y}}{\longrightarrow} 2 n\left\langle v_{1} \mid J v_{3}\right\rangle\left\langle v_{4} \mid v_{6}\right\rangle\left\langle v_{5} \mid J v_{7}\right\rangle \sum_{j, k, l=1}^{2 n} e_{j} \otimes e_{k} \otimes J f_{k} \otimes f_{j} \otimes v_{2} \otimes e_{l} \otimes f_{l}
\end{aligned}
$$

which is the image under $\rho$ of $2 n$ times the following marked diagram:

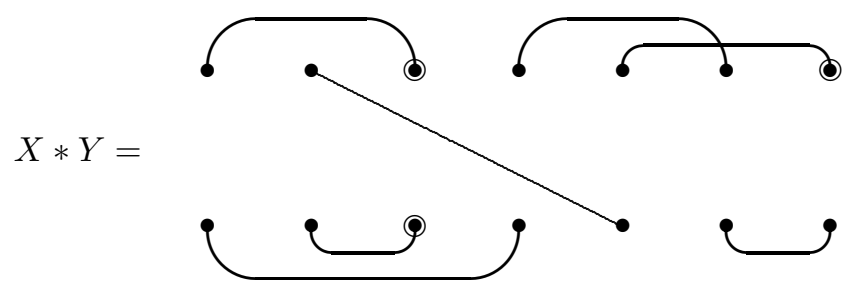

Let us consider another example:

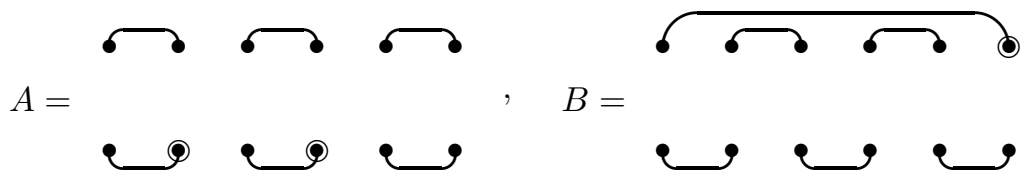

Then

$$
v_{1} \otimes \cdots \otimes v_{6} \stackrel{\rho_{A}}{\longrightarrow}\left\langle v_{1} \mid v_{2}\right\rangle\left\langle v_{3} \mid v_{4}\right\rangle\left\langle v_{5} \mid v_{6}\right\rangle \sum_{a, b, c=1}^{2 n} e_{a} \otimes J f_{a} \otimes e_{b} \otimes J f_{b} \otimes e_{c} \otimes f_{c}
$$

while

$$
\begin{aligned}
e_{a} \otimes J f_{a} & \otimes e_{b} \otimes J f_{b} \otimes e_{c} \otimes f_{c} \\
& \stackrel{\rho_{B}}{\longrightarrow}\left\langle e_{a} \mid J f_{c}\right\rangle\left\langle J f_{a} \mid e_{b}\right\rangle\left\langle J f_{b} \mid e_{c}\right\rangle \sum_{j, k, l=1}^{2 n} e_{j} \otimes f_{j} \otimes e_{k} \otimes f_{k} \otimes e_{l} \otimes f_{l} .
\end{aligned}
$$


Now,

$$
\begin{aligned}
& \sum_{a, b, c=1}^{2 n}\left\langle e_{a} \mid J f_{c}\right\rangle\left\langle J f_{a} \mid e_{b}\right\rangle\left\langle J f_{b} \mid e_{c}\right\rangle=-\sum_{a, b, c=1}^{2 n}\left\langle e_{a} \mid J f_{c}\right\rangle\left\langle f_{a} \mid J e_{b}\right\rangle\left\langle J f_{b} \mid e_{c}\right\rangle \\
& =-\sum_{b, c=1}^{2 n}\left\langle\sum_{a=1}^{2 n}\left\langle e_{a} \mid J f_{c}\right\rangle f_{a} \mid J e_{b}\right\rangle\left\langle J f_{b} \mid e_{c}\right\rangle=-\sum_{b, c=1}^{2 n}\left\langle J f_{c} \mid J e_{b}\right\rangle\left\langle J f_{b} \mid e_{c}\right\rangle \\
& =-\sum_{b, c=1}^{2 n}\left\langle f_{c} \mid e_{b}\right\rangle\left\langle J f_{b} \mid e_{c}\right\rangle=-\sum_{c=1}^{2 n}\left\langle J\left(\sum_{b=1}^{2 n}\left\langle e_{b} \mid f_{c}\right\rangle f_{b}\right) \mid e_{c}\right\rangle \\
& =-\sum_{c=1}^{2 n}\left\langle J f_{c} \mid e_{c}\right\rangle=0
\end{aligned}
$$

because of the skew-symmetry of $J$ and since $\sum_{c=1}^{2 n} e_{c} \otimes f_{c}=\sum_{c=1}^{2 n} f_{c} \otimes e_{c}$ (this element of $\left(V_{\mathbb{R}}\right) \otimes_{\mathbb{R}}\left(V_{\mathbb{R}}\right)$ does not depend on the chosen dual bases). Therefore $\rho_{A} \rho_{B}=0$.

The previous arguments show the general rule to multiply marked diagrams:

Given two marked diagrams $X$ and $Y$, draw $Y$ below $X$ and connect the $l^{\text {th }}$ upper vertex of $Y$ with the $l^{\text {th }}$ lower vertex of $X$, to get a 'marked graph' $G(X, Y)$. For the previously considered marked diagrams $X$ and $Y$, we have:

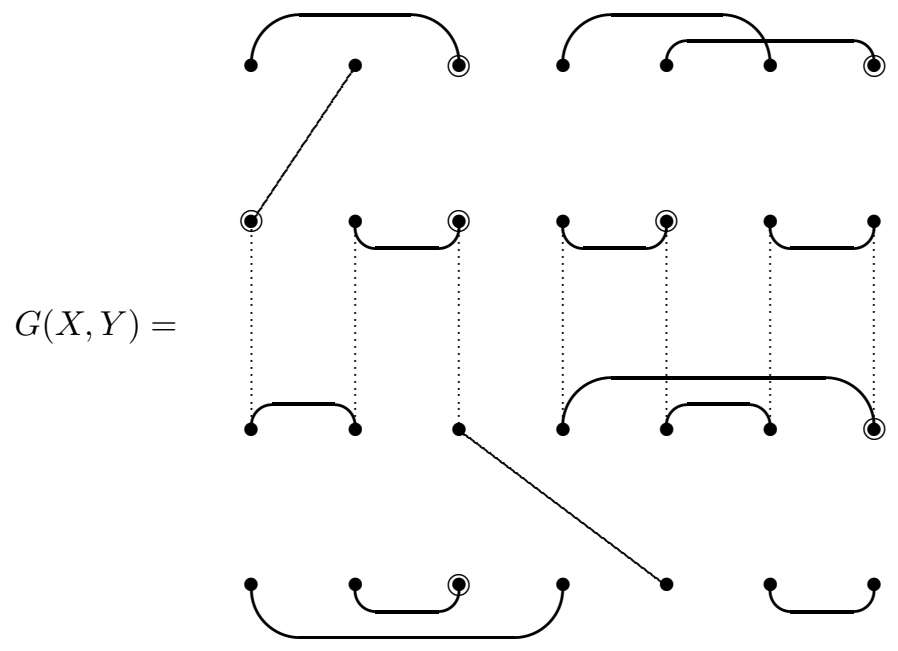

Then

$$
X Y=\gamma(X, Y) X * Y,
$$

where $\gamma(X, Y) \in \mathbb{R}$ is defined below and $X * Y$ is the marked diagram whose vertices are the vertices in the upper row of $X$ and the vertices in the lower row of $Y$ with the horizontal edges that appear in these rows. The rightmost vertices of these horizontal edges inherit the marking in $X$ or $Y$. Moreover, there is a vertical edge joining any upper vertex of $X$ with a lower vertex of $Y$ precisely if there is a path in $G(X, Y)$ joining these vertices. The lowermost vertex of any of these vertical edges is marked if and only if there is an odd number of marked vertices along the corresponding path in $G(X, Y)$. (For the example above, $X * Y$ appears in (4.3).) 
Besides,

(1) Let $p$ be any path in $G(X, Y)$, we move the (say) $s$ marks on the vertices along $p$ to the bottommost vertex and define

$$
\gamma(p)=(-1)^{\text {number of horizontal moves }}(-1)^{\left\lfloor\frac{s}{2}\right\rfloor}
$$

$(\lfloor x\rfloor$ is the largest integer $\leq x)$. Thus, for instance, consider the path in the previous example:

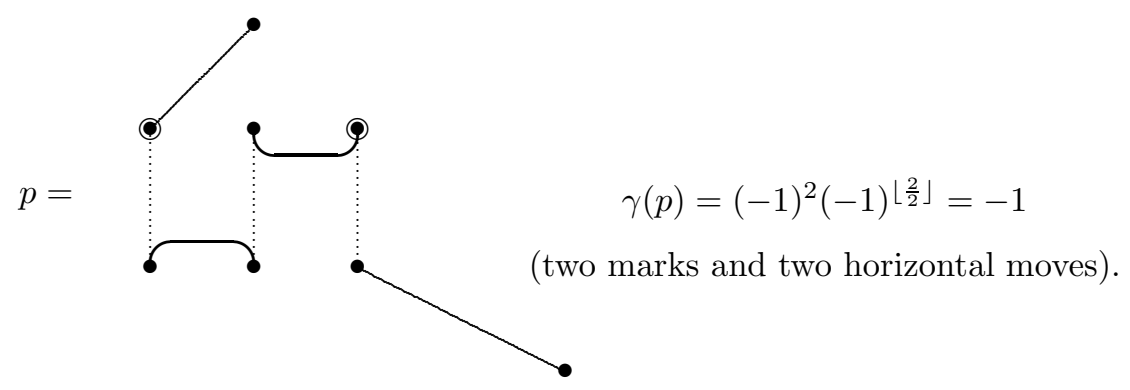

(2) Let $l$ be any loop in $G(X, Y)$, fix any vertex in $l$ and move all the marks, say $s$, in the vertices of $l$ to this fixed vertex. Then define

$$
\gamma(l)= \begin{cases}0 & \text { if } s \text { is odd, } \\ (-1)^{\text {number of horizontal moves }}(-1)^{\frac{s}{2}} & \text { if } s \text { is even. }\end{cases}
$$

For instance, taking the loop of the previous example:

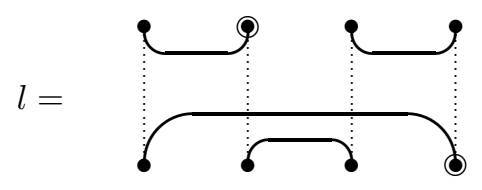

$$
\gamma(l)=(-1)^{2}(-1)^{\frac{2}{2}}=-1 .
$$

The definitions of $\gamma(p)$ and $\gamma(l)$ are made so as to take into account the skewsymmetry of $J$ and the fact that $J^{2}=-1$.

Finally, define

$$
\gamma(X, Y)=(2 n)^{\text {number of loops in } G(X, Y)} \prod_{\substack{p \text { path } \\ \text { in } G(X, Y)}} \gamma(p) \prod_{\substack{l \text { loop } \\ \text { in } G(X, Y)}} \gamma(l) .
$$

The resulting algebra (over the real field) thus defined over $\mathrm{D}_{\mathrm{r}}^{\text {marked }}$ will be denoted by $\mathrm{D}_{\mathrm{r}}^{\text {marked }}(n)$.

Proposition 4.6. $\mathrm{D}_{\mathrm{r}}^{\text {marked }}(n)$ is an associative algebra for any $r, n \in \mathbb{N}$.

Proof. The product in $\mathrm{D}_{\mathrm{r}}^{\text {marked }}(n)$ is defined in such a way as to ensure that $\rho$ : $\mathrm{D}_{\mathrm{r}}^{\text {marked }}(n) \rightarrow \operatorname{End}_{U(V, h)}\left(\otimes_{\mathbb{R}}^{r}\left(V_{\mathbb{R}}\right)\right)$ is a homomorphism of algebras. Hence the result is obvious for $n \geq r$ by Proposition 4.1 In general, formulas (4.4) and (4.5) show that for $X, Y \in \mathrm{D}_{\mathrm{r}}^{\text {marked }}$,

$$
X Y=(2 n)^{l(X, Y)} s(X, Y) X * Y,
$$

where $l(X, Y) \in \mathbb{Z}_{\geq 0}$ is the number of loops in $G(X, Y)$ and $s(X, Y) \in\{0,1,-1\}$. Both $l(X, Y)$ and $s(X, Y)$ are independent of $n$. The associativity of $\mathrm{D}_{\mathrm{r}}^{\text {marked }}(n)$ is 
equivalent to the validity of $(X Y) Z=X(Y Z)$ for any marked diagrams $X, Y, Z$, or to the validity of

$$
(2 n)^{l(X, Y)+l(X * Y, Z)} s(X, Y) s(X * Y, Z)=(2 n)^{l(Y, Z)+l(X, Y * Z)} s(Y, Z) s(X, Y * Z)
$$

which is satisfied if and only if for any marked diagrams $X, Y, Z$

$$
\left\{\begin{array}{l}
l(X, Y)+l(X * Y, Z)=l(Y, Z)+l(X, Y * Z), \\
s(X, Y) s(X * Y, Z)=s(Y, Z) s(X, Y * Z),
\end{array}\right.
$$

which does not depend on $n$. Therefore $\mathrm{D}_{\mathrm{r}}^{\operatorname{marked}}(n)$ is associative if and only if so is $\mathrm{D}_{\mathrm{r}}^{\operatorname{marked}}(m)$ for $m$ large enough, which is indeed the case.

Remark 4.7. The proof above suggests the consideration of the algebra $\mathrm{D}_{\mathrm{r}}^{\operatorname{marked}}(x)$ over $\mathbb{R}(x)$, with a basis formed by the marked diagrams and multiplication given by

$$
X Y=x^{l(X, Y)} s(X, Y) X * Y
$$

for any marked diagrams $X, Y$; in analogy with the Brauer algebras $\operatorname{Br}_{r}(x)$ considered in [19, 9].

Remark 4.8. Given an edge of a marked diagram, call it positive if its bottommost or rightmost vertex is not marked, and negative otherwise. Hence the marked diagrams can be identified with the signed diagrams in [14, 15. The algebra $\mathrm{D}_{\mathrm{r}}^{\operatorname{marked}}(x)$ is then defined over the same vector space as the Signed Brauer Algebra defined in these references, although the multiplication is different.

Let us now proceed to give a presentation of $\mathrm{D}_{\mathrm{r}}^{\text {marked }}(x)$ by generators and relations. We will assume $r \geq 4$, the situation for $r \leq 3$ is simpler and can be deduced easily along the same lines. First, let us consider the following marked diagrams:

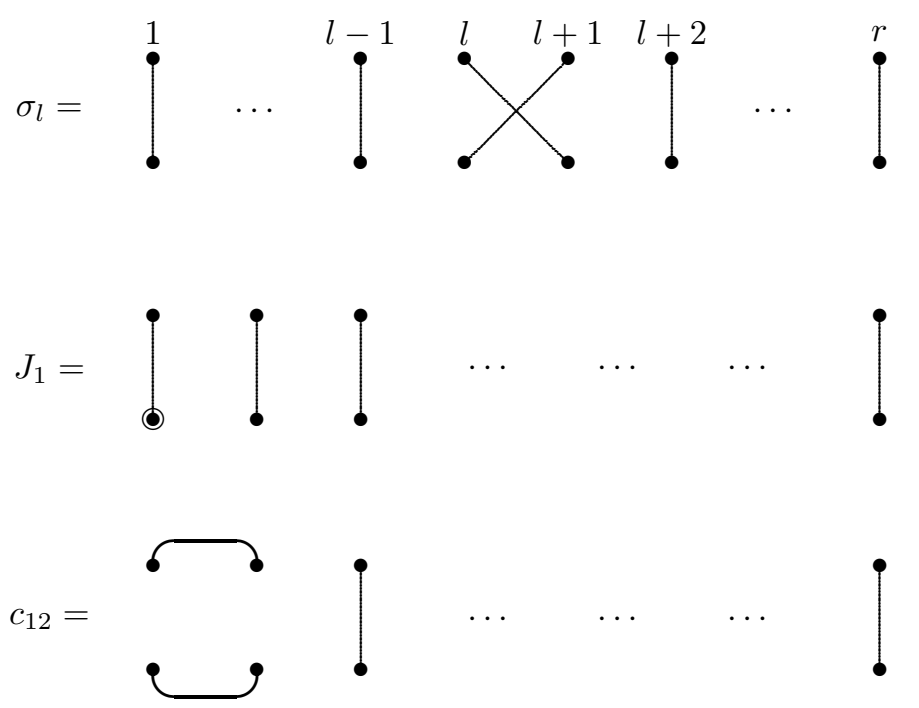

These generate the algebra $\mathrm{D}_{\mathrm{r}}^{\text {marked }}(x)$, because the $\sigma_{l}$ 's generate the symmetric group, $\sigma J_{1} \sigma^{-1}=J_{\sigma(l)}$ and $\sigma c_{12} \sigma^{-1}=c_{\sigma(1) \sigma(2)}$ for any $\sigma$ in the symmetric group.

Then the following relations among these elements are easily checked:

(i) $\sigma_{l}^{2}=1,1 \leq l \leq r-1$, 
(ii) $\sigma_{l} \sigma_{m}=\sigma_{m} \sigma_{l}, 1 \leq l, m \leq r-1,|l-m| \geq 2$,

(iii) $\left(\sigma_{l} \sigma_{l+1}\right)^{3}=1,1 \leq l \leq r-2$,

(iv) $J_{1}^{2}=-1$,

(v) $J_{1} \sigma_{l}=\sigma_{l} J_{1}, 2 \leq l \leq r-1$,

(vi) $\left(J_{1} \sigma_{1}\right)^{4}=1$,

(vii) $c_{12}^{2}=x c_{12}$,

(viii) $c_{12} \sigma_{l}=\sigma_{l} c_{12}, 3 \leq l \leq r-1$,

(ix) $c_{12} \sigma_{1}=c_{12}=\sigma_{1} c_{12}$,

(x) $c_{12} \sigma_{2} c_{12}=c_{12}$,

(xi) $c_{12} J_{1} c_{12}=0$,

(xii) $\left(\sigma_{1} J_{1}+J_{1}\right) c_{12}=0=c_{12}\left(J_{1}+J_{1} \sigma_{1}\right)$,

(xiii) $\sigma_{2} \sigma_{1} J_{1} \sigma_{1} \sigma_{2} c_{12}=c_{12} \sigma_{2} \sigma_{1} J_{1} \sigma_{1} \sigma_{2}$,

(xiv) $c_{12} \sigma_{2} \sigma_{1} \sigma_{3} \sigma_{2} c_{12} \sigma_{2} \sigma_{3} \sigma_{1} \sigma_{2}=\sigma_{2} \sigma_{1} \sigma_{3} \sigma_{2} c_{12} \sigma_{2} \sigma_{3} \sigma_{1} \sigma_{2} c_{12}$.

Notice that (vi) is equivalent to $J_{1} J_{2}=J_{2} J_{1}$, (xii) to $J_{1} c_{12}=-J_{2} c_{12}$ and $c_{12} J_{1}=-c_{12} J_{2}$, (xiii) to $J_{3} c_{12}=c_{12} J_{3}$ and (xiv) to $c_{12} c_{34}=c_{34} c_{12}$.

Take the free associative algebra $D$ over $\mathbb{R}(x)$ generated by elements $\sigma_{1}, \ldots, \sigma_{r-1}$, $J_{1}, c_{12}$, subject to the relations (i)-(xiv) above. $\mathrm{D}_{\mathrm{r}}^{\operatorname{marked}}(x)$ is a quotient of this algebra, and to show that they are isomorphic it is enough to check that the dimension of $D$ is $2^{r}((2 r-1) ! !)$. To do so, first the subalgebra generated by the $\sigma_{l}$ 's is (isomorphic to) the group algebra of the symmetric group $S_{r}$ (in principle it is a quotient of the group algebra, but the corresponding subalgebra of $\mathrm{D}_{\mathrm{r}}^{\operatorname{marked}}(x)$ is the whole group algebra). Moreover, define recursively in $D$ the new elements $J_{l+1}=\sigma_{l} J_{l} \sigma_{l}$, $1 \leq l \leq r-2$. Because of (v) one has $\sigma J_{l} \sigma^{-1}=J_{\sigma(l)}$ for any $\sigma \in S_{r} \subseteq D$. Then relation (vi) yields $J_{1} J_{2}=J_{2} J_{1}$ and with this one easily proves that $J_{l} J_{m}=J_{m} J_{l}$ for any $l, m$, and that the subalgebra of $D$ generated by the $\sigma_{l}$ 's and $J_{1}$ is the span of the elements $J_{\mathcal{P}} \sigma$, where $\mathcal{P} \subseteq\{1, \ldots, r\}, \sigma \in S_{r}$ and $J_{\mathcal{P}}=\prod_{p \in P} J_{p}\left(J_{\emptyset}=1\right)$.

Now define in $D$ the elements $c_{p q}(p \neq q)$ by $c_{p q}=\sigma c_{12} \sigma^{-1}$, where $\sigma \in S_{r}$ satisfies $\sigma(1)=p, \sigma(2)=q$. This is well defined by the relations in (viii) and, because of (ix), $c_{p q}=c_{q p}$ for any $p, q$. Then relation (xii) is equivalent to $J_{3} c_{12}=c_{12} J_{3}$ which, by conjugation with suitable elements of $S_{r}$, yields $J_{s} c_{p q}=c_{p q} J_{s}$ for different $s, p, q$. Also, relation (xiv) becomes $c_{12} c_{34}=c_{34} c_{12}$, and again, by conjugation, it yields $c_{p q} c_{p^{\prime} q^{\prime}}=c_{p^{\prime} q^{\prime}} c_{p q}$ for different $p, q, p^{\prime}, q^{\prime}$. Finally, for distinct elements $p, q, q^{\prime}, c_{p q} c_{p q^{\prime}}=c_{p q}\left(q q^{\prime}\right) c_{p q}\left(q q^{\prime}\right)=c_{p q}\left(q q^{\prime}\right)=\left(q q^{\prime}\right) c_{p q^{\prime}}$, thanks to relation (x) and its conjugates. Besides, $c_{p q} J_{p} c_{p q}=0$ by (xi), while $c_{p q} J_{p} c_{p q^{\prime}}=-c_{p q} J_{q^{\prime}} c_{p q^{\prime}}=$ $-J_{q^{\prime}} c_{p q} c_{p q^{\prime}}$, and also $c_{p q} J_{p} c_{p q^{\prime}}=-c_{p q} J_{q} c_{p q^{\prime}}=-c_{p q} c_{p q^{\prime}} J_{q}$ for different $p, q, q^{\prime}$. With all these relations, any word in the generators belongs to the linear span of the elements

$$
J_{\mathcal{Q}} c_{p_{1} q_{1}} \cdots c_{p_{s} q_{s}} J_{\mathcal{P} c} \sigma
$$

where $p_{1}<\cdots<p_{s}, q_{1}, \ldots, q_{s}$ are different elements in $\{1, \ldots, r\}, \mathcal{Q} \subseteq\left\{q_{1}, \ldots, q_{s}\right\}$, $\mathcal{P}^{c} \subseteq\{1, \ldots, r\} \backslash\left\{p_{1}, \ldots, p_{s}\right\}$ and $\sigma \in S_{r}$. But $c_{p q}(p q)=c_{p q}$ by relation (ix) and $c_{p q} J_{q}(p q)=c_{p q}(p q) J_{p}=c_{p q} J_{p}=-c_{p q} J_{q}$, so one can assume that $\sigma\left(p_{l}\right)<\sigma\left(q_{l}\right)$ for any $l=1, \ldots, s$. With this extra condition on $\sigma$ in (4.9), each element in (4.9) is in bijection with a unique marked diagram.

This finishes the proof of:

Theorem 4.10. Assuming $r \geq 4, \mathrm{D}_{\mathrm{r}}^{\text {marked }}(x)$ is the associative algebra over $\mathbb{R}(x)$ generated by $\left\{\sigma_{1}, \ldots, \sigma_{r-1}, J_{1}, c_{12}\right\}$, subject to the relations (i)-(xiv). 
Remark 4.11. For $r=3$, it is enough to consider relations (i), (iii), (iv), (vi), (vii), and (ix)-(xiii); while for $r=2$ (i), (iv), (vi), (vii), (ix), (xi) and (xii) are sufficient.

\section{Decomposition into IRREDUCiBles}

In this section, the hermitian form $h$ on $V$ will be assumed to be positive definite, so $U(V, h) \cong U(n)$, the compact unitary group (see however Remark 5.4). The goal here is to use the results in the previous sections, together with [2] (see also [18), to decompose $\otimes_{\mathbb{R}}^{r}\left(V_{\mathbb{R}}\right)$ into a direct sum of irreducible $U(V, h)$-modules.

Let us first recall [,,$\S 26.3]$ that given a real Lie group $G$, a representation of $G$ on a complex vector space $W$ is said to be real if it comes from a representation of $G$ on a real vector space $W_{0}$ by extension of scalars (up to isomorphism, $W=\mathbb{C} \otimes_{\mathbb{R}} W_{0}$ ). This is equivalent to saying that there is a conjugate linear endomorphism of $W$ whose square is the identity. Note also that in this case $W_{\mathbb{R}}=W_{0} \oplus i W_{0}$ is the direct sum of two copies of the $G$-module $W_{0}$. The representation is said to be quaternionic if there is a conjugate linear endomorphism whose square is minus the identity and complex if it is neither real nor quaternionic.

In case $W$ is irreducible as a representation over $\mathbb{C}$, that is, in case it has no proper complex subspaces invariant under $G, W$ is real if and only if $W_{\mathbb{R}}$ contains a proper irreducible submodule $W_{0}$. Thus, as a $G$-module, $W_{\mathbb{R}}$ is isomorphic to the direct sum of two copies of $W_{0}$ and its centralizer algebra $\operatorname{End}_{G}\left(W_{\mathbb{R}}\right)$ is isomorphic to the algebra of matrices $\operatorname{Mat}_{2}(\mathbb{R})$. On the other hand, if $W$ is complex or quaternionic, then $W_{\mathbb{R}}$ remains irreducible and $\operatorname{End}_{G}\left(W_{\mathbb{R}}\right)$ is isomorphic either to $\mathbb{C}$ or to $\mathbb{H}$, respectively.

To achieve the goal of this section, first an element $p \in\{1, \ldots, r\}$ is fixed, and for simplicity we will take $p=1$. Then, from Proposition 2.2, Corollary 2.4, Proposition 2.6 and (2.9)

$$
\begin{aligned}
\otimes_{\mathbb{R}}^{r}\left(V_{\mathbb{R}}\right) & =\bigoplus_{1 \in \mathcal{P} \subseteq\{1, \ldots, r\}}\left(\otimes_{\mathbb{R}}^{r}\left(V_{\mathbb{R}}\right)\right) e_{\mathcal{P}} \\
& \cong \bigoplus_{q=1}^{r}\left(\begin{array}{c}
r \\
q
\end{array}\right)\left(\left(\otimes_{\mathbb{C}}^{q} V\right) \otimes_{\mathbb{C}}\left(\otimes_{\mathbb{C}}^{r-q} V^{*}\right)\right)_{\mathbb{R}}
\end{aligned}
$$

and, as complex vector spaces,

$$
\left[\otimes_{\mathbb{R}}^{r}\left(V_{\mathbb{R}}\right)\right]_{\mathbb{C}, 1} \cong \bigoplus_{q=1}^{r}\left(\begin{array}{l}
r \\
q
\end{array}\right)\left(\left(\otimes_{\mathbb{C}}^{q} V\right) \otimes_{\mathbb{C}}\left(\otimes_{\mathbb{C}}^{r-q} V^{*}\right)\right) .
$$

Therefore the module (over $\mathbb{C}$ ) $V_{q, r-q}:=\left(\otimes_{\mathbb{C}}^{q} V\right) \otimes_{\mathbb{C}}\left(\otimes_{\mathbb{C}}^{r-q} V^{*}\right)$ has to be decomposed into a direct sum of irreducible $U(V, h)$-modules. Let us think in terms of the associated Lie algebra $\mathfrak{u}(V, h)$, which is a form of the general linear Lie algebra $\mathfrak{g l}(V)$. The irreducible $\mathfrak{u}(V, h)$-submodules of $V_{q, r-q}$ over $\mathbb{C}$ are exactly the irreducible $\mathfrak{g l}(V)$-submodules, and these are determined in [18] and [2]: the irreducible $\mathfrak{g l}(V)$ submodules of $V_{q, r-q}$ are in one-to-one correspondence with the pairs $(\tau, L)$ where:

(1) $L=\left[\left(m_{1}, m_{1}^{\prime}\right), \ldots,\left(m_{s}, m_{s}^{\prime}\right)\right]$ is a sequence of pairs with $1 \leq m_{1}<m_{2}<$ $\cdots<m_{s} \leq q, m_{1}^{\prime}, \ldots, m_{s}^{\prime}$ are different elements in $\{q+1, \ldots, r\} \quad(s \leq$ $\min \{q, r-q\}) . L$ indicates the slots where a contraction is made among $V$ and $V^{*}$.

(2) $\tau=\left(\tau^{+}, \tau^{-}\right)$is a pair of standard rational tableaux, where $\tau^{+}$(respectively $\tau^{-}$) is obtained by filling the boxes in a Young frame with the numbers in 
$\{1, \ldots, q\} \backslash\left\{m_{1}, \ldots, m_{s}\right\}$ (resp. in $\{q+1, \ldots, r\} \backslash\left\{m_{1}^{\prime}, \ldots, m_{s}^{\prime}\right\}$ ). Being standard means that the numbers strictly increase from left to right across each row and from top to bottom in each column.

(3) If $\operatorname{dim} V=n<r$, an extra technical condition has to be satisfied (see [2] Theorem 1.11]) that, in particular, forces the sum of the number of rows in $\tau^{+}$and $\tau^{-}$to be at most $n$.

Example. $r=7, q=3, L=[(1,6),(2,4)], \tau^{+}=3, \tau^{-}=$\begin{tabular}{l}
5 \\
\hline 7
\end{tabular} .

If we fix a basis of $V$, so that $\mathfrak{g l}(V) \cong \mathfrak{g l}_{n}$, the complex Lie algebra of $n \times n$ matrices, and consider the Cartan subalgebra $\mathfrak{h}$ formed by the diagonal matrices, let $\epsilon_{l} \in \mathfrak{h}^{*}(l=1, \ldots, n)$ be given by $\epsilon_{l}\left(\operatorname{diag}\left(\alpha_{1}, \ldots, \alpha_{n}\right)\right)=\alpha_{l}$. Then the highest weight of the irreducible module associated to a pair $(\tau, L)$ as above is $\lambda_{1} \epsilon_{1}+$ $\cdots+\lambda_{n} \epsilon_{n}$, where $\lambda_{1} \geq \lambda_{2} \geq \cdots \geq \lambda_{t}>0$ are the lengths of the rows of $\tau^{+}$, while $-\lambda_{n} \geq-\lambda_{n-1} \geq \cdots \geq-\lambda_{n-t^{\prime}+1}>0$ are the lengths of the rows of $\tau^{-}$and $\lambda_{t+1}=\cdots=\lambda_{n-t^{\prime}}=0$.

Once $V_{q, r-q}$ is decomposed into a direct sum of irreducible modules for $U(V, h)$ over $\mathbb{C}$, what is left to be done is to check which of these modules remain irreducible as modules for $U(V, h)$ over $\mathbb{R}$ (that is, the representation is either complex or quaternionic) and which of them do not (the representation is real):

\section{Proposition 5.1.}

(1) For $r \neq 2 q$, the irreducible $U(V, h)$-submodules of $\left(\otimes_{\mathbb{C}}^{q} V\right) \otimes_{\mathbb{C}}\left(\otimes_{\mathbb{C}}^{r-q} V^{*}\right)$ are all complex, so they remain irreducible as modules over $\mathbb{R}$.

(2) The same happens if $r=2 q$ for the irreducible $U(V, h)$-modules of $\left(\otimes_{\mathbb{C}}^{q} V\right) \otimes_{\mathbb{C}}$ $\left(\otimes_{\mathbb{C}}^{q} V^{*}\right)$ which correspond to pairs $(\tau, L)$, where the Young frames of $\tau^{+}$and $\tau^{-}$are different.

(3) The irreducible $U(V, h)$-submodules of $\left(\otimes_{\mathbb{C}}^{q} V\right) \otimes_{\mathbb{C}}\left(\otimes_{\mathbb{C}}^{q} V^{*}\right)$ over $\mathbb{C}$ which correspond to pairs $(\tau, L)$, with equal Young frames of $\tau^{+}$and $\tau^{-}$are real, so they split into a direct sum of two copies of an irreducible $U(V, h)$-module over $\mathbb{R}$.

Proof. If $q \neq r-q$ (in particular, if $r$ is odd), then $i 1 \in \mathfrak{u}(V, h)$ acts as

$$
(q-(r-q)) i 1=(2 q-r) i 1 \neq 0
$$

on $V_{q, r-q}=\left(\otimes_{\mathbb{C}}^{q} V\right) \otimes_{\mathbb{C}}\left(\otimes_{\mathbb{C}}^{r-q} V^{*}\right)$, and hence the action of scalar multiplication by imaginary complex numbers is "included" in the action of $\mathfrak{u}(V, h)$. Thus all the irreducible submodules of $V_{q, r-q}$ over $\mathbb{C}$ are complex, so they are irreducible as modules over $\mathbb{R}$.

If $q=r-q$, the argument above shows that the action of $i 1 \in \mathfrak{u}(V, h)$ on $V_{q, q}(r-q=q)$ is trivial, so we have to consider only the action of $\mathfrak{s u}(V, h)$. Hence, the highest weights of the irreducible $\mathfrak{g l}(V)$-submodules in $V_{q, q}$ are of the form $\lambda_{1} \epsilon_{1}+\cdots+\lambda_{n} \epsilon_{n}$, with $\lambda_{1} \geq \cdots \geq \lambda_{n}$ and $\lambda_{1}+\cdots+\lambda_{n}=0$, so that $\lambda_{1} \epsilon_{1}+\cdots+\lambda_{n} \epsilon_{n}=\left(\lambda_{1}-\lambda_{2}\right) \omega_{1}+\cdots+\left(\lambda_{n-1}-\lambda_{n}\right) \omega_{n-1}$, where $\omega_{1}=\epsilon_{1}, \omega_{2}=$ $\epsilon_{1}+\epsilon_{2}, \ldots, \omega_{n-1}=\epsilon_{1}+\cdots+\epsilon_{n-1}$, are the fundamental dominant weights of $\mathfrak{s l}(V)$. Notice that the integers $\lambda_{1}-\lambda_{2}, \ldots, \lambda_{n-1}-\lambda_{n}$, together with the condition $\lambda_{1}+\cdots+\lambda_{n}=0$, determine $\lambda_{1}, \ldots, \lambda_{n}$. The conditions for this highest weight to 
yield a real representation are [6, Proposition 26.24]:

$$
\begin{aligned}
& \lambda_{l}-\lambda_{l+1}=\lambda_{n-l}-\lambda_{n-l+1}, 1 \leq l \leq n-1, \\
& n \text { odd, or } n=4 k, \text { or } n=4 k+2 \text { and } \lambda_{2 k+1}-\lambda_{2 k+2} \text { even, }
\end{aligned}
$$

while the conditions to yield a quaternionic representation are (5.2) and $n=4 k+2$ and $\lambda_{2 k+1}-\lambda_{2 k+2}$ is odd. But (5.2) is equivalent to $\lambda_{l}+\lambda_{n+1-l}(1 \leq l \leq n-1)$ being constant, which together with the condition $\lambda_{1}+\cdots+\lambda_{n}=0$ yields $\lambda_{l}+\lambda_{n+1-l}=0$, which implies $\lambda_{2 k+1}-\lambda_{2 k+2}=2 \lambda_{2 k+1}$ for $n=4 k+2$. Therefore, the condition (5.3) follows from (5.2), and condition (5.2) is equivalent to the restriction of the Young frames of both $\tau^{+}$and $\tau^{-}$being the same, as required.

As a first example, consider $\left(V_{\mathbb{R}}\right) \otimes_{\mathbb{R}}\left(V_{\mathbb{R}}\right)$, which splits as

$$
\begin{aligned}
\left(V_{\mathbb{R}}\right) \otimes_{\mathbb{R}}\left(V_{\mathbb{R}}\right) & =\left(\left(V_{\mathbb{R}}\right) \otimes_{\mathbb{R}}\left(V_{\mathbb{R}}\right)\right) e_{1} \oplus\left(\left(V_{\mathbb{R}}\right) \otimes_{\mathbb{R}}\left(V_{\mathbb{R}}\right)\right) e_{2} \\
& \simeq\left(V \otimes_{\mathbb{C}} V\right)_{\mathbb{R}} \oplus\left(V \otimes_{\mathbb{C}} V^{*}\right)_{\mathbb{R}} \\
& \simeq S^{2}(V)_{\mathbb{R}} \oplus \Lambda^{2}(V)_{\mathbb{R}} \oplus \mathbb{C}_{\mathbb{R}} \oplus \mathfrak{s l}(V)_{\mathbb{R}},
\end{aligned}
$$

where $e_{1}=\frac{1}{2}\left(1-J_{1} J_{2}\right), e_{2}=\frac{1}{2}\left(1+J_{1} J_{2}\right)$. Here $S^{2}(V)_{\mathbb{R}}$ (the symmetric tensors) and $\Lambda^{2}(V)_{\mathbb{R}}$ (skew-symmetric tensors) are irreducible as $U(V, h)$-modules over $\mathbb{R}$, while $\mathbb{C}_{\mathbb{R}}$ is a direct sum of two trivial one-dimensional modules over $\mathbb{R}$, and $\mathfrak{s l}(V)_{\mathbb{R}}=$ $\mathfrak{s u}(V, h) \oplus i \mathfrak{s u}(V, h)$ is a direct sum of two copies of $\mathfrak{s u}(V, h)$.

In the remaining part of this paper, we will consider the motivating example of Gray and Hervella [7] considered in the Introduction, as well as another related example by Abbena and Garbiero [1].

Example (Gray-Hervella, 1978 [7]). Since $V_{\mathbb{R}} \cong\left(V_{\mathbb{R}}\right)^{*}$ as modules for $U(V, h)$, the problem described in the Introduction amounts to decompose

$$
\begin{aligned}
W & =\left\{x \in \otimes_{\mathbb{R}}^{3}\left(V_{\mathbb{R}}\right): x(23)=-x=x J_{2} J_{3}\right\} \\
& =\left\{x \in\left(\otimes_{\mathbb{R}}^{3}\left(V_{\mathbb{R}}\right)\right) \frac{1}{2}\left(1-J_{2} J_{3}\right): x(23)=-x\right\}
\end{aligned}
$$

into a direct sum of irreducible submodules for $U(V, h)$. First notice that

$$
\begin{aligned}
\frac{1}{2}\left(1-J_{2} J_{3}\right) & =\frac{1}{2}\left(1-J_{2} J_{3}\right) \frac{1}{2}\left(1-J_{1} J_{3}+1+J_{1} J_{3}\right) \\
& =e_{\{1,2,3\}}+e_{\{2,3\}}
\end{aligned}
$$

(a sum of orthogonal idempotents) in the notation of Section 2. Hence, by Proposition 2.6

$$
\begin{aligned}
\left(\otimes_{\mathbb{R}}^{3}\left(V_{\mathbb{R}}\right)\right) \frac{1}{2}\left(1-J_{2} J_{3}\right) & =\left(\otimes_{\mathbb{R}}^{3}\left(V_{\mathbb{R}}\right)\right) e_{\{1,2,3\}} \oplus\left(\otimes_{\mathbb{R}}^{3}\left(V_{\mathbb{R}}\right)\right) e_{\{2,3\}} \\
& \cong\left(V \otimes_{\mathbb{C}} V \otimes_{\mathbb{C}} V\right)_{\mathbb{R}} \oplus\left(V^{*} \otimes_{\mathbb{C}} V \otimes_{\mathbb{C}} V\right)_{\mathbb{R}}
\end{aligned}
$$

and from this isomorphism, it immediately follows that

$$
W \cong\left(V \otimes_{\mathbb{C}} \Lambda^{2}(V)\right)_{\mathbb{R}} \oplus\left(V^{*} \otimes_{\mathbb{C}} \Lambda^{2}(V)\right)_{\mathbb{R}},
$$

and it is enough to decompose each one of these two summands into irreducible $\mathfrak{g l}(V)$-modules.

The first summand is $\left(V \otimes_{\mathbb{C}} V \otimes_{\mathbb{C}} V\right) \frac{1}{2}(1-(23))$ and, since in the group algebra $\mathbb{C} S_{3}$ the idempotent $\frac{1}{2}(1-(23))=e_{T_{1}}+e_{T_{2}}$ is the sum of two orthogonal primitive 
idempotents, where

$$
T_{1}=\begin{array}{|l|}
\hline 2 \\
\hline 3 \\
\hline 1
\end{array}, \quad T_{2}=\begin{array}{|l|l|}
\hline 2 & 1 \\
\hline 3 & \\
\hline
\end{array}
$$

(that is, $e_{T_{1}}=\frac{1}{6} \sum_{\sigma \in S_{3}}(-1)^{\sigma} \sigma$ and $e_{T_{2}}=\frac{1}{3}(1+(12))(1-(23))$ ), it follows that $V \otimes_{\mathbb{C}} \Lambda^{2}(V)=\left(\otimes_{\mathbb{C}}^{3} V\right) e_{T_{1}} \oplus\left(\otimes_{\mathbb{C}}^{3} V\right) e_{T_{2}}$ (direct sum of two irreducible modules if $\operatorname{dim} V \geq 3$ ), which under the isomorphisms correspond to

$$
\begin{aligned}
& W_{1}=\left\{x \in \otimes_{\mathbb{R}}^{3}\left(V_{\mathbb{R}}\right): x J_{1}=x J_{2}=x J_{3}, x e_{T_{1}}=x\right\}, \\
& W_{2}=\left\{x \in \otimes_{\mathbb{R}}^{3}\left(V_{\mathbb{R}}\right): x J_{1}=x J_{2}=x J_{3}, x e_{T_{2}}=x\right\} .
\end{aligned}
$$

But $x e_{T_{2}}=x$ if and only if $x(23)=-x$ and $x(1+(123)+(132))=0$. For any $x$ satisfying these two conditions, one checks easily that the condition $x J_{1}=x J_{2}$ follows from $x J_{2}=x J_{3}$. Hence

$$
W_{2}=\{x \in W: x(1+(123)+(132))=0\},
$$

and, similarly,

$$
W_{1}=\{x \in W: x(12)=-x\} .
$$

On the other hand, assuming $\operatorname{dim} V \geq 3, V^{*} \otimes_{\mathbb{C}} \Lambda^{2}(V)$ decomposes [2] into:

- $\operatorname{ker} c$, where $c: V^{*} \otimes_{\mathbb{C}} \Lambda^{2}(V) \rightarrow V, f \otimes(u \otimes v-v \otimes u) \mapsto f(u) v-f(v) u$, which corresponds to the pair $(\tau, L)$, with $\tau=\left(\frac{1}{2}, 3\right)$ and $L=\emptyset$.

- $\left\{\sum_{l=1}^{n} u_{l}^{*} \otimes\left(u_{l} \otimes v-v \otimes u_{l}\right): v \in V\right\} \cong V$, where $\left\{u_{l}\right\}_{l=1}^{n}$ and $\left\{u_{l}^{*}\right\}_{l=1}^{n}$ are dual bases of $V$ and $V^{*}$. This is a 'diagonal' submodule of the ones that correspond to the pairs $(\tau, L)$ with $L=[(1,3)]$ and $\tau=(2, \emptyset)$ and with $L=[(2,3)]$ and $\tau=(1, \emptyset)$.

Under the isomorphisms, these submodules correspond to:

$$
\begin{aligned}
& W_{3}=\left\{x \in W: x J_{1}=-x J_{2} \text { and } x c_{12}=0\right\}, \\
& W_{4}=\left\{\sum_{l=1}^{2 n}\left(e_{l} \otimes f_{l} \otimes v\right) \frac{1}{4}\left(1+J_{1} J_{3}\right)(1-(23): v \in V\},\right.
\end{aligned}
$$

where the $e_{l}$ 's and $f_{l}$ 's constitute dual bases of $V_{\mathbb{R}}$ relative to $\langle\mid\rangle$.

The situation for $\operatorname{dim} V \leq 2$ is simpler.

We recover in this way the decomposition given in [7].

Example (Abbena-Garbiero, 1988 [1]). One has to decompose

$$
\begin{aligned}
K & =\left\{x \in \otimes_{\mathbb{R}}^{3}\left(V_{\mathbb{R}}\right): x(23)=-x=-x J_{2} J_{3}\right\} \\
& =\left\{x \in\left(\otimes_{\mathbb{R}}^{3}\left(V_{\mathbb{R}}\right)\right) \frac{1}{2}\left(1+J_{2} J_{3}\right): x(23)=-x\right\} .
\end{aligned}
$$

As before, $\frac{1}{2}\left(1+J_{2} J_{3}\right)=e_{\{1,2\}}+e_{\{1,3\}}$, so

$$
\left(\otimes_{\mathbb{R}}^{3} V\right) \frac{1}{2}\left(1+J_{2} J_{3}\right) \cong\left(V \otimes_{\mathbb{C}} V \otimes_{\mathbb{C}} V^{*}\right)_{\mathbb{R}} \oplus\left(V \otimes_{\mathbb{C}} V^{*} \otimes_{\mathbb{C}} V\right)_{\mathbb{R}}
$$

by means of the isomorphism $\Phi$ given by

$$
\Phi\left(\left(v_{1} \otimes v_{2} \otimes v_{3}\right) \frac{1}{2}\left(1+J_{2} J_{3}\right)\right)=v_{1} \otimes v_{2} \otimes h\left(-, v_{3}\right)+v_{1} \otimes h\left(-, v_{2}\right) \otimes v_{3} .
$$


Now, the following diagram is commutative:

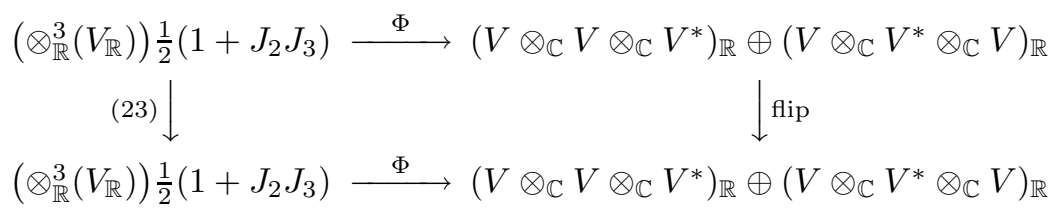

Here on the left we have the right action of the transposition (23), while on the right $\left(v_{1} \otimes v_{2} \otimes f\right)$ flip $=v_{1} \otimes f \otimes v_{2}$ and $\left(v_{1} \otimes f \otimes v_{2}\right)$ flip $=v_{1} \otimes v_{2} \otimes f$ for any $v_{1}, v_{2} \in V$ and $f \in V^{*}$.

Therefore, the linear map given by

$$
\begin{gathered}
K=\left(\otimes_{\mathbb{R}}^{3}\left(V_{\mathbb{R}}\right)\right) \frac{1}{4}\left(1+J_{2} J_{3}\right)(1-(23)) \longrightarrow\left(V \otimes_{\mathbb{C}} V \otimes_{\mathbb{C}} V^{*}\right)_{\mathbb{R}} \\
\left(v_{1} \otimes v_{2} \otimes v_{3}\right) \frac{1}{4}\left(1+J_{2} J_{3}\right)(1-(23)) \mapsto \frac{1}{2}\left(v_{1} \otimes v_{2} \otimes h\left(-, v_{3}\right)-\right. \\
\left.v_{1} \otimes v_{3} \otimes h\left(-, v_{2}\right)\right)
\end{gathered}
$$

is an isomorphism of $U(V, h)$-modules.

If $\operatorname{dim} V \geq 3, V \otimes_{\mathbb{C}} V \otimes_{\mathbb{C}} V^{*}$ decomposes into the direct sum of the irreducible $\mathfrak{g l}(V)$-modules corresponding to the pairs $(\tau, L)$ in the following list (see [2]):

$$
\begin{aligned}
& \tau=\left(\begin{array}{l|l}
\hline 1 & 2 \\
\hline
\end{array}, 3\right), \quad L=\emptyset, \\
& \tau=\left(\begin{array}{l}
1,3 \\
\hline 2
\end{array}, 3\right), \quad L=\emptyset, \\
& \tau=(1, \emptyset), \quad L=[(2,3)], \\
& \tau=(2, \emptyset), \quad L=[(1,3)] .
\end{aligned}
$$

With $c: V \otimes_{\mathbb{C}} V \otimes_{\mathbb{C}} V^{*} \rightarrow V, v_{1} \otimes v_{2} \otimes f \mapsto f\left(v_{1}\right) v_{2}$, the first two modules correspond to $\left(S^{2}(V) \otimes_{\mathbb{C}} V^{*}\right) \cap \operatorname{ker} c$ and $\left(\Lambda^{2}(V) \otimes_{\mathbb{C}} V^{*}\right) \cap \operatorname{ker} c$, while the last two modules are isomorphic to $V$. One recovers from here the decomposition given in 1]. The details are left to the reader.

Remark 5.4. The argument used in the proof of Proposition 5.1 for the case $r \neq 2 q$ remains valid for any nondegenerate hermitian form. Therefore the restriction of $h$ being definite can be relaxed to being nondegenerate in the previous examples.

\section{ACKNOWLEDGEMENTS}

The author is indebted to Pedro Martínez Gadea, who introduced him to the problems in 7, 1] and sent a copy of [5], to Georgia Benkart for very illuminating conversations about this work, and to the anonymous referee for many comments that have improved the exposition.

\section{REFERENCES}

[1] E. Abbena and S. Garbiero, Almost Hermitian homogeneous structures, Proc. Edinburgh Math. Soc. (2) 31 (1988), no. 3, 375-395. MR 90c:53119

[2] G. Benkart, M. Chakrabarti, T. Halverson, R. Leduc, C. Lee, and J. Stroomer, Tensor product representations of general linear groups and their connections with Brauer algebras, J. Algebra 166 (1994), no. 3, 529-567. MR 95d:20071

[3] J.S. Birman and H. Wenzl, Braids, link polynomials and a new algebra, Trans. Amer. Math. Soc. 313 (1989), no. 1, 249-273. MR 90g:57004 
[4] R. Brauer, On algebras which are connected with semisimple Lie groups, Ann. of Math. 38 (1937), 857-872.

[5] P. Fortuny, and P. Martínez Gadea, On the classification theorems of almost-Hermitian or homogeneous Kähler structures, to appear in Rocky Mountain. J. Math.

[6] W. Fulton and J. Harris, Representation theory. A first course, Graduate Texts in Mathematics, vol. 129, Springer-Verlag, New York, 1991, Readings in Mathematics. MR 93a:20069

[7] A. Gray and L.M. Hervella, The sixteen classes of almost Hermitian manifolds and their linear invariants, Ann. Mat. Pura Appl. (4) 123 (1980), 35-58. MR 81m:53045

[8] T. Halverson and A. Ram, Characters of algebras containing a Jones basic construction: the Temperley-Lieb, Okasa, Brauer, and Birman-Wenzl algebras, Adv. Math. 116 (1995), no. 2, 263-321. MR 96k:16023

[9] P. Hanlon and D. Wales, On the decomposition of Brauer's centralizer algebras, J. Algebra 121 (1989), no. 2, 409-445. MR 91a:2004la

[10] N. Iwahori, Some remarks on tensor invariants of $\mathrm{O}(n), \mathrm{U}(n), \operatorname{Sp}(n)$, J. Math. Soc. Japan 10 (1958), 145-160. MR 23:A1722

[11] M. Jimbo, A $q$-analogue of $U(\mathfrak{g l}(N+1))$, Hecke algebra, and the Yang-Baxter equation, Lett. Math. Phys. 11 (1986), no. 3, 247-252. MR 87k:17011

[12] R. Leduc and A. Ram, A ribbon Hopf algebra approach to the irreducible representations of centralizer algebras: the Brauer, Birman-Wenzl, and type A Iwahori-Hecke algebras, Adv. Math. 125 (1997), no. 1, 1-94. MR 98c:20015

[13] J. Murakami, The representations of the $q$-analogue of Brauer's centralizer algebras and the Kauffman polynomial of links, Publ. Res. Inst. Math. Sci. 26 (1990), no. 6, 935-945. MR 91m:57004

[14] M. Parvathi and M. Kamaraj, Signed Brauer's algebras, Comm. Algebra 26 (1998), no. 3, 839-855. MR 99c:16028

[15] M. Parvathi and C. Selvaraj, Signed Brauer's algebras as centralizer algebras, Comm. Algebra 27 (1999), no. 12, 5985-5998. MR 2000j:16051

[16] I. Schur, Über eine Klasse von Matrixen die sich einer gegebenen Matrix zuordnen lassen. Thesis, Berlin, 1901. Reprinted in Gesammelte Abhandlungen, Band I, Springer-Verlag, Berlin, 1973, Herausgegeben von Alfred Brauer und Hans Rohrbach. MR 57:2858a

[17] _ Über die rationalen Darstellungen der allgemeinen linearen Gruppe. 1927. Reprinted in Gesammelte Abhandlungen, Band III, Springer-Verlag, Berlin, 1973, Herausgegeben von Alfred Brauer und Hans Rohrbach. MR 57:2858c

[18] J.R. Stembridge, Rational tableaux and the tensor algebra of $\mathrm{gl}_{n}, \mathrm{~J}$. Combin. Theory Ser. A 46 (1987), no. 1, 79-120. MR 89a:05012

[19] H. Wenzl, On the structure of Brauer's centralizer algebras, Ann. of Math. (2) 128 (1988), no. 1, 173-193. MR 89h:20059

[20] H. Weyl, The Classical Groups. Their Invariants and Representations, Princeton University Press, Princeton, N.J., 1939. MR 1:42c

Departamento de Matemáticas, Universidad de Zaragoza, 50009 Zaragoza, Spain

E-mail address: elduque@unizar.es 